\title{
Article \\ Metabolomics Comparison of Drug-Resistant and Drug-Susceptible Pseudomonas aeruginosa Strain (Intra- and Extracellular Analysis)
}

\author{
Karolina Anna Mielko ${ }^{1}$, Sławomir Jan Jabłoński ${ }^{2}$, tukasz Pruss ${ }^{1,3}{ }^{\circledR}$, Justyna Milczewska ${ }^{4}\left(\mathbb{D}\right.$, Dorota Sands ${ }^{4}$, \\ Marcin Lukaszewicz ${ }^{2}$ and Piotr Młynarz ${ }^{1, *(\mathbb{D})}$ \\ 1 Department of Biochemistry, Molecular Biology and Biotechnology, Faculty of Chemistry, Wroclaw University \\ of Science and Technology, 50-373 Wroclaw, Poland; karolina.mielko@pwr.edu.pl (K.A.M.); \\ lukasz.pruss@pwr.edu.pl (Ł.P.) \\ 2 Biotransformation Department, Faculty of Biotechnology, University of Wroclaw, 50-383 Wroclaw, Poland; \\ slawomir.jablonski@uwr.edu.pl (S.J.J.); marcin.lukaszewicz@uwr.edu.pl (M.Ł.) \\ 3 Ardigen, 30-394 Kraków, Poland \\ 4 Cystic Fibrosis Department, Institute of Mother and Child, 01-211 Warsaw, Poland; \\ justyna.milczewska@imid.med.pl (J.M.); dorota.sands@imid.med.pl (D.S.) \\ * Correspondence: piotr.mlynarz@pwr.edu.pl
}

check for updates

Citation: Mielko, K.A.; Jabłoński, S.J.; Pruss, Ł.; Milczewska, J.; Sands, D.; Łukaszewicz, M.; Młynarz, P. Metabolomics Comparison of Drug-Resistant and Drug-Susceptible Pseudomonas aeruginosa Strain (Intraand Extracellular Analysis). Int. J. Mol. Sci. 2021, 22, 10820. https:// doi.org/10.3390/ijms221910820

Academic Editor: Giovanna Batoni

Received: 24 August 2021

Accepted: 2 October 2021

Published: 6 October 2021

Publisher's Note: MDPI stays neutral with regard to jurisdictional claims in published maps and institutional affiliations.

Copyright: (c) 2021 by the authors. Licensee MDPI, Basel, Switzerland. This article is an open access article distributed under the terms and conditions of the Creative Commons Attribution (CC BY) license (https:/ / creativecommons.org/licenses/by/ $4.0 /)$

\begin{abstract}
Pseudomonas aeruginosa is a common human pathogen belonging to the ESKAPE group The multidrug resistance of bacteria is a considerable problem in treating patients and may lead to increased morbidity and mortality rate. The natural resistance in these organisms is caused by the production of specific enzymes and biofilm formation, while acquired resistance is multifactorial. Precise recognition of potential antibiotic resistance on different molecular levels is essential. Metabolomics tools may aid in the observation of the flux of low molecular weight compounds in biochemical pathways yielding additional information about drug-resistant bacteria. In this study, the metabolisms of two P. aeruginosa strains were compared-antibiotic susceptible vs. resistant. Analysis was performed on both intra- and extracellular metabolites. The ${ }^{1} \mathrm{H}$ NMR method was used together with multivariate and univariate data analysis, additionally analysis of the metabolic pathways with the FELLA package was performed. The results revealed the differences in P. aeruginosa metabolism of drug-resistant and drug-susceptible strains and provided direct molecular information about $P$. aeruginosa response for different types of antibiotics. The most significant differences were found in the turnover of amino acids. This study can be a valuable source of information to complement research on drug resistance in P. aeruginosa.
\end{abstract}

Keywords: Pseudomonas aeruginosa; metabolomics; antibiotic resistance; NMR spectroscopy

\section{Introduction}

Pseudomonas aeruginosa is a Gram-negative opportunistic human pathogen [1], which causes infections in chronic wounds and in the urinary tract. Moreover, it is responsible for respiratory tract infections in cystic fibrosis $(\mathrm{CF})$, obstructive lung disease, or mechanically ventilated patients [2]. As a part of the ESKAPE pathogens group (Enterococcus faecium, Staphylococcus aureus, Klebsiella pneumoniae, Acinetobacter baumannii, Pseudomonas aeruginosa, and Enterobacter species), P. aeruginosa is a significant cause of nosocomial infections. In some $P$. aeruginosa strains, antibiotic therapy is not successful, despite its sensitivity in laboratory tests [3,4]. Due to the increasing number of multidrug-resistant (MDR) isolates, the WHO has recognized P. aeruginosa as a priority pathogen for antibiotic research [5]. The development of new antibacterial therapies and understanding antibiotic resistance mechanisms is crucial for clinical practice and finding new treatment possibilities [6-8].

Multidrug resistance of $P$. aeruginosa relies on several mechanisms: (i) antibiotic molecules may be neutralized by specialized enzymes ( $\beta$-lactams, aminoglycosides), 
(ii) therapeutic compounds may also be removed from the cell by efflux pumps ( $\beta$-lactams, quinolones, and some aminoglycosides), (iii) alteration of the antibiotic target molecule, (iv) modifications in the penicillin-binding proteins (PBPs), and (v) changes in OPrD porin [4,9-12].

An important factor affecting drug efficiency is drug accessibility for targeted microorganisms. Biofilm produced by P. aeruginosa is a physical barrier protecting bacteria from antibiotics [13]. Some P. aeruginosa strains exhibit decreased expression of genes encoding the porins (outer membrane channel proteins) used by certain types of antibiotics (e.g., rifamycins and quinolones) to enter the cell [11]. Antibiotic intake may also be reduced due to reduced membrane potential [14]. These P. aeruginosa attributes are important factors that should be considered during the development of an efficient antibiotic therapy.

Bacterial cells can become antibiotic-resistant when stringent response (SR) is activated due to the nutrient limitation resulting in reduced bacterial multiplication. The correct activity of SR is also crucial for biofilm development and stability [15].

The drug resistance phenomenon is analyzed on many levels: genomic, transcriptomic, proteomic, and metabolomics. The last link of this omics chain may provide information on antibiotic resistance mechanisms [16]. Metabolomics is focused on the analysis and monitoring of low molecular weight compounds involved in cell metabolism. Metabolomic studies performed on microorganisms so far usually rely on chromatographic techniques coupled with mass spectrometry (MS) and nuclear magnetic resonance spectroscopy (NMR) [17,18].

Metabolomics experiments have enabled the identification of new metabolic pathways [18], the recognition of bacterial strain origin [19], the identification of microorganism species [20,21], and the analysis of the influence of different external factors on bacteria [22]. Moreover, metabolomic and genomic analysis helped explain P. aeruginosa polymyxin resistance [23]. Metabolome analysis techniques are also considered an advanced diagnostic tool for bacterial infections [24].

The presence of some antibiotic resistance mechanisms may be correlated with changes in metabolite concentration (inside and outside the cell). Resistance to some antibiotics is associated with the presence and activity of proteins involved in the processing of certain metabolites in the cell (oprD protein required for carbapenem uptake is an amino acid transporter [10] and activator of SR, which results in changes in the expression of the enzymes involved in the main metabolic pathways including amino acid synthesis [25]). Metabolome analysis would give additional information alongside antibiotic sensitivity tests. This may help in the choice of appropriate antibiotic therapy, as well as enabling research in new therapeutic strategies [26].

The metabolomics comparison of strains with different antibiotic resistance could give information about the differences in the phenotype of both strains and may show the direction of further (more detailed) investigations. The analysis of intra- and extracellular metabolites allows us to observe the differences in intracellular machinery of the bacteria, but also the association with the bacterial environment. All these reasons can give additional information for transcriptomics and genomics studies and may enable the recognition of more antibiotic resistance mechanisms in P. aeruginosa. This study aimed to delineate metabolic differences between two P. aeruginosa strains isolated from CF patients: a strain resistant to the majority of available antibiotics (except colistin and ciprofloxacin) and an antibiotic susceptible strain, by use of metabolomics tools using the ${ }^{1} \mathrm{H}$ NMR method together with univariate and multivariate analysis of intra- and extracellular metabolites and the application of bioinformatics metabolic pathway analysis software (FELLA).

\section{Results}

\subsection{Antibiotic Resistance Test}

The results of antibiotic resistance tests are presented in Table 1. In this experiment, antibiotics from the following groups: aminoglycoside (disrupting protein synthesis by binding to the $30 \mathrm{~S}$ ribosomal subunit), beta-lactams (disrupting peptidoglycan biosynthesis), quinolone (disrupting DNA replication), and polymyxin (disrupting cell membrane) 
were used. Strain PAW17 was susceptible to all tested antibiotics. Strain PAW23 was susceptible only to two antibiotics: ciprofloxacin and colistin.

Table 1. Antibiotic resistance of Pseudomonas aeruginosa strains (S—susceptible, R—resistant).

\begin{tabular}{cccc}
\hline Antibiotic Name & Class & PAW17 & PAW23 \\
\hline amikacin & aminoglycoside & $\mathrm{S}$ & $\mathrm{R}$ \\
gentamicin & aminoglycoside & $\mathrm{S}$ & $\mathrm{R}$ \\
netilmicin & aminoglycoside & $\mathrm{S}$ & $\mathrm{R}$ \\
tobramycin & aminoglycoside & $\mathrm{S}$ & $\mathrm{R}$ \\
imipenem & beta-lactam & $\mathrm{S}$ & $\mathrm{R}$ \\
meropenem & beta-lactam & $\mathrm{S}$ & $\mathrm{R}$ \\
piperacillin & beta-lactam & $\mathrm{S}$ & $\mathrm{R}$ \\
piperacillin/tazobactam & beta-lactam & $\mathrm{S}$ & $\mathrm{R}$ \\
ticarcillin/clavulanic acid & beta-lactam & $\mathrm{S}$ & $\mathrm{R}$ \\
ceftazidime & beta-lactam & $\mathrm{S}$ & $\mathrm{R}$ \\
cefepime & beta-lactam & $\mathrm{S}$ & $\mathrm{R}$ \\
ciprofloxacin & quinolone & $\mathrm{S}$ & $\mathrm{S}$ \\
levofloxacin & quinolone & $\mathrm{S}$ & $\mathrm{R}$ \\
colistin & polymyxin (peptide) & $\mathrm{S}$ & $\mathrm{S}$ \\
\hline
\end{tabular}

\subsection{Metabolites Identification}

\subsubsection{Intracellular Metabolites}

In total, 32 intracellular metabolites were identified (5-aminopentanoate, acetate, adenine, alanine, AMP, aspartate, betaine, ethanol, formate, glucose, glutamate, glycine, histamine, histidine, homoserine, isobutyrate, isocitrate, isoleucine, lactate, leucine, methionine, NAD+, oxypurinol, phenylalanine, pyruvate, sarcosine, succinate, threonine, tyrosine, UMP, uracil, and valine). Information about the chemical shift for each metabolite is available in Supplementary Materials (Table S1). The set of identified metabolites was identical for both strains.

The representative ${ }^{1} \mathrm{H}$ NMR spectrum of intracellular metabolites is presented below (Figure 1).

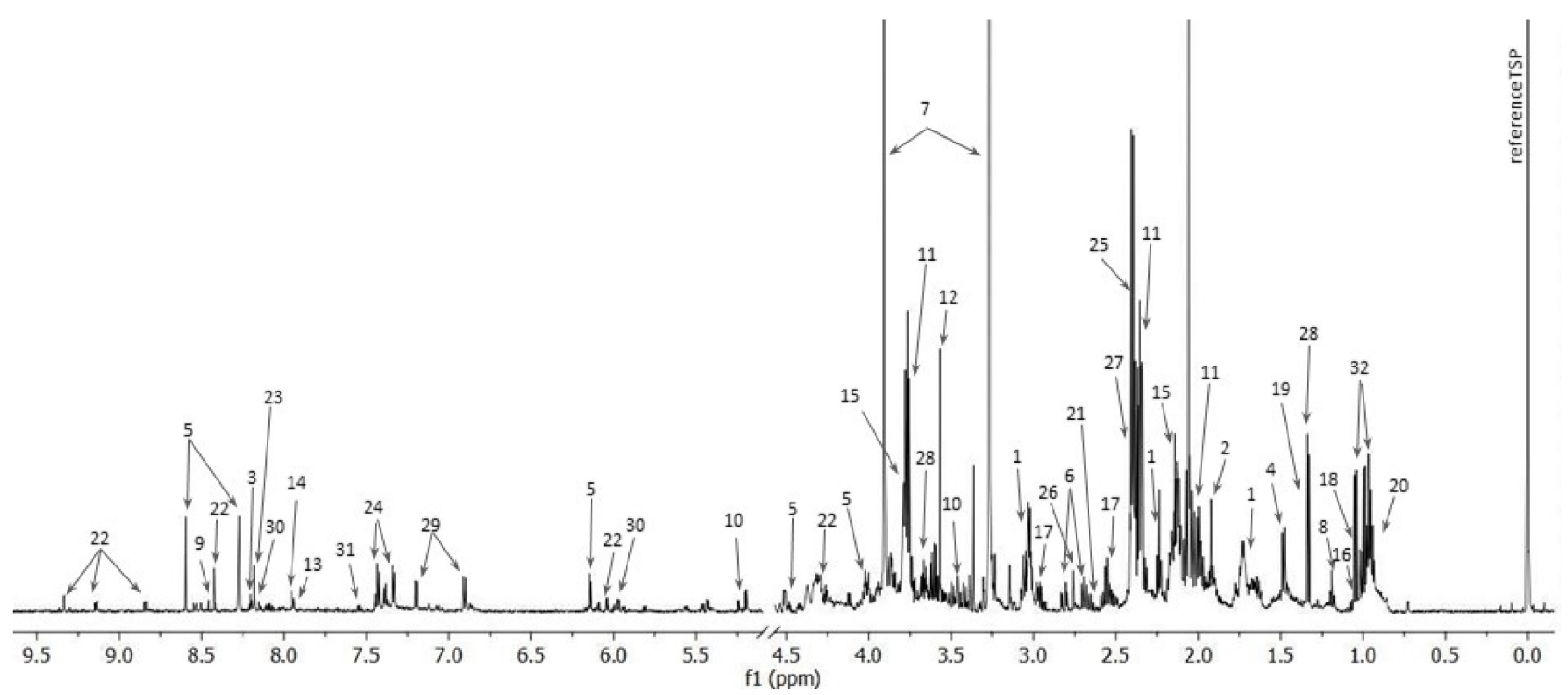

Figure 1. The representative $1 \mathrm{D}^{1} \mathrm{H}$ NMR spectrum of intracellular metabolites of drug-susceptible Pseudomonas aeruginosa strain. (1-5-aminopentanoate, 2-acetate, 3-adenine, 4-alanine, 5-AMP, 6-aspartate, 7-betaine, 8-ethanol, 9formate, 10-glucose, 11 -glutamate, 12-glycine, 13-histamine, 14-histidine, 15-homoserine, 16-isobutyrate, 17isocitrate, 18-isoleucine, 19-lactate, 20-leucine, 21-methionine, 22-NAD+, 23-oxypurinol, 24-phenylalanine, 25-pyruvate, 26-sarcosine, 27-succinate, 28-threonine, 29-tyrosine, 30-UMP, 31-uracil, 32-valine). 


\subsubsection{Intracellular Metabolites}

In total, 27 extracellular metabolites were identified (6-hydroxynicotinate, acetate, alanine, betaine, formate, glycine, histamine, histidine, imidazole, isobutyrate, glutamate, aspartate, asparagine, pyroglutamate, isoleucine, leucine, lysine, methanol, methionine, oxypurinol, phenylalanine, pyruvate, threonine, trehalose, tryptophan, tyrosine, and valine). Four metabolites were not present in the post-culture medium (glutamate, aspartate, asparagine, and pyroglutamate). Information about the chemical shift for each metabolite is available in Supplementary Materials (Table S2).

The representative ${ }^{1} \mathrm{H}$ NMR spectrum of intracellular metabolites is presented in (Figure 2).

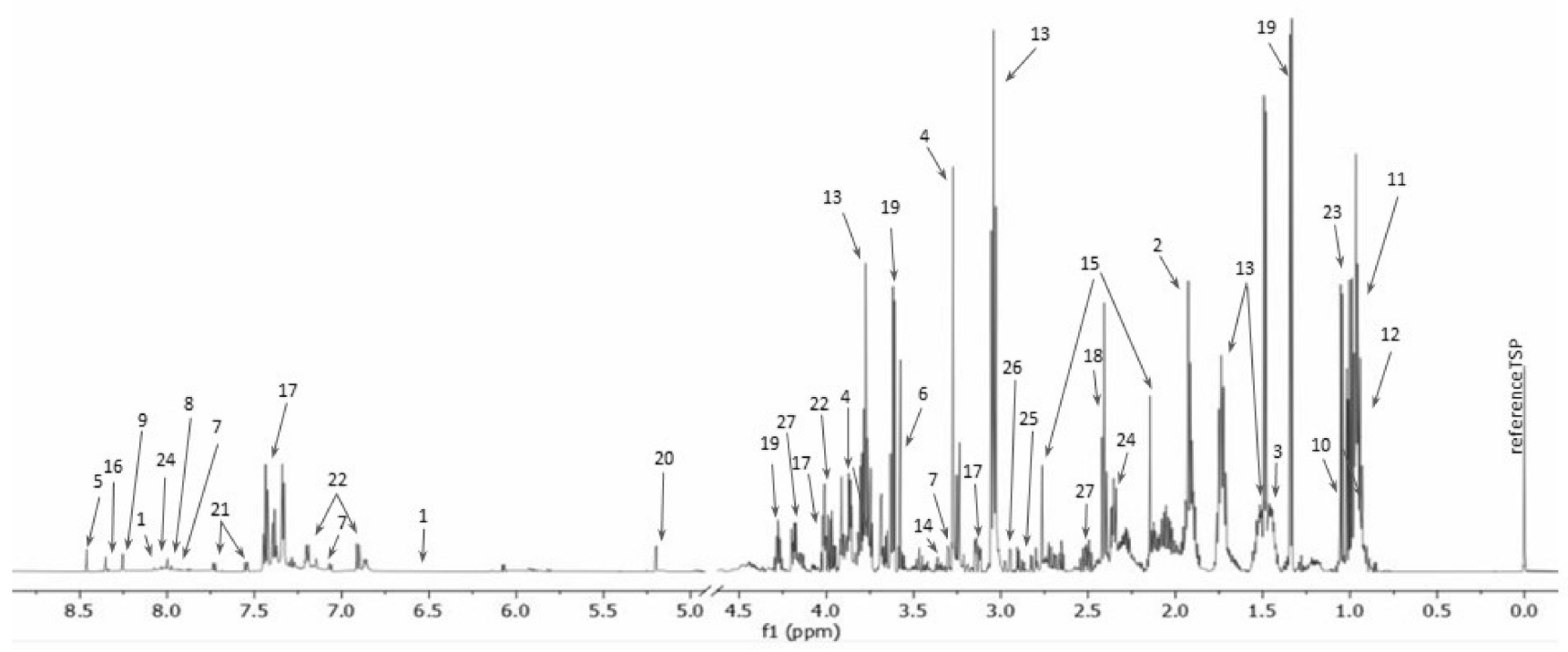

Figure 2. The representative $1 \mathrm{D}{ }^{1} \mathrm{H}$ NMR spectrum of extracellular metabolites of drug-susceptible Pseudomonas aeruginosa strain (1-6-hydroxynicotinate; 2-acetate; 3-alanine; 4-betaine; 5-formate; 6-glycine; 7-histamine; 8-histidine; 9-imidazole; 10-isobutyrate; 11—isoleucine; 12-leucine; 13-lysine; 14-methanol; 15-methionine; 16-oxypurinol; 17-phenylalanine; 18-pyruvate; 19—threonine; 20—trehalose; 21—tryptophan; 22—tyrosine; 23-valine; 24-glutamate; 25-aspartate; 26-asparagine; 27-pyroglutamate).

The total number of intra- and extracellular metabolites identified was 39. Among these, 19 metabolites were common to intra- and extracellular environments (histidine, aspartate, glutamate, histamine, alanine, pyruvate, valine, isoleucine, leucine, betaine, methionine, formate, glycine, threonine, phenylalanine, tyrosine, isobutyrate, oxypurinol, and acetate); 13 metabolites were identified in cell extracts (succinate, homoserine, lactate, UMP, sarcosine, ethanol, isocitrate, adenine, glucose, NAD+, AMP, 5-aminopentanoate, and uracil) and eight were identified only in the culture medium (imidazole, asparagine, methanol, 6-hydroxynicotinate, pyroglutamate, tryptophan, lysine, and trehalose).

\subsection{Multivariate Data Analysis}

The performed PCA-multivariate unsupervised analysis between drug-resistant and drug-susceptible isolates revealed the natural grouping between bacterial intra- and extracellular metabolites (Figure 3). Direct comparison of intracellular metabolites revealed a more similar metabolomics profile than between extracellular metabolites. The first three principal components (PC) accounted respectively for $84.7 \%, 8.74 \%$, and $2.56 \%$ of the total variance in the data $(\mathrm{R} 2 \mathrm{X}=0.998)$. The obtained loading plots analysis showed three metabolites differentiated between bacteria cells and medium, which are: glycine, betaine, and pyruvate. 

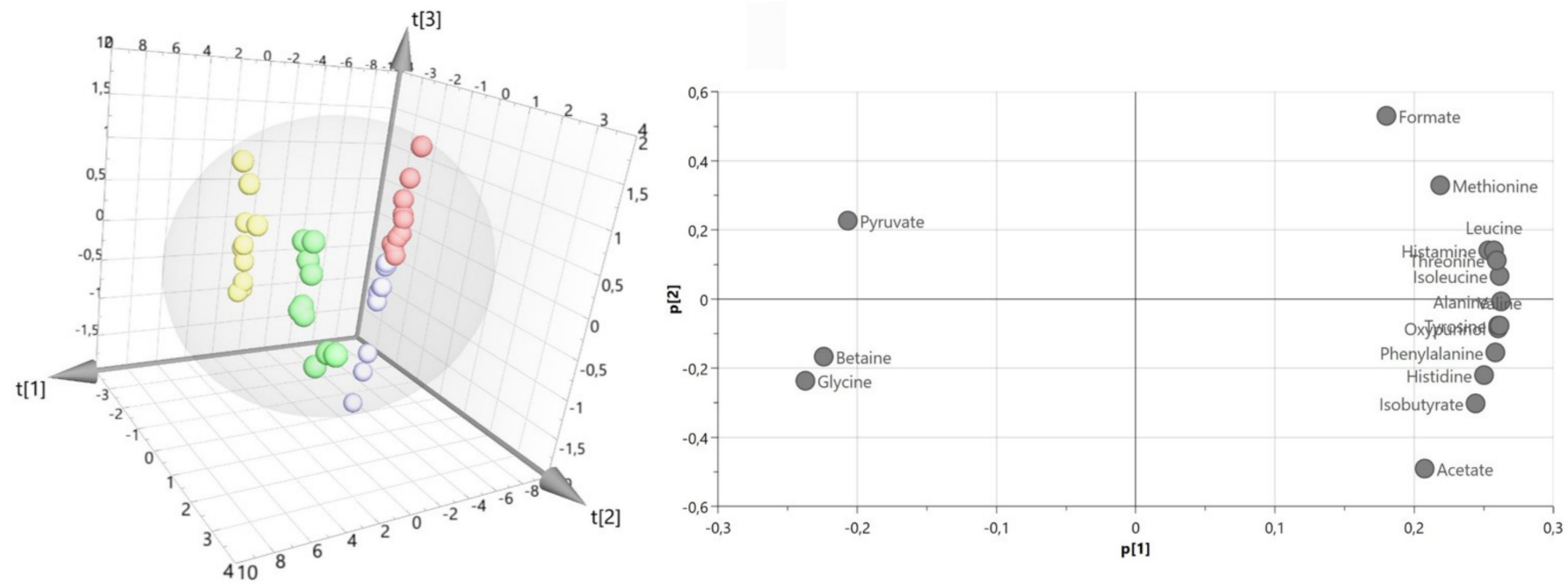

Figure 3. PCA model plot and corresponding loading plot for of ${ }^{1} \mathrm{H}$ NMR data of all metabolites of P. aeruginosa strains (drug-resistant extracellular (green), drug-susceptible extracellular (yellow), drug-resistant intracellular (blue), and drugsusceptible intracellular (red)). Symbols in the same color represent biological repetitions.

Supervised analysis OPLS-DA analysis provides the strain's grouping. The CVANOVA test of this model gave statistically important results (model with parameters are available in Supplementary Materials (Figure S1)).

\subsubsection{Intracellular Metabolites}

PCA score plot revealed the clustering of bacterial isolates with and without antibiotic resistance (Figure 4). The first and second principal components (PC) accounted, respectively, for $41.6 \%$ and $21.5 \%$ of the total variance in the data $(R 2 X=0.631)$.
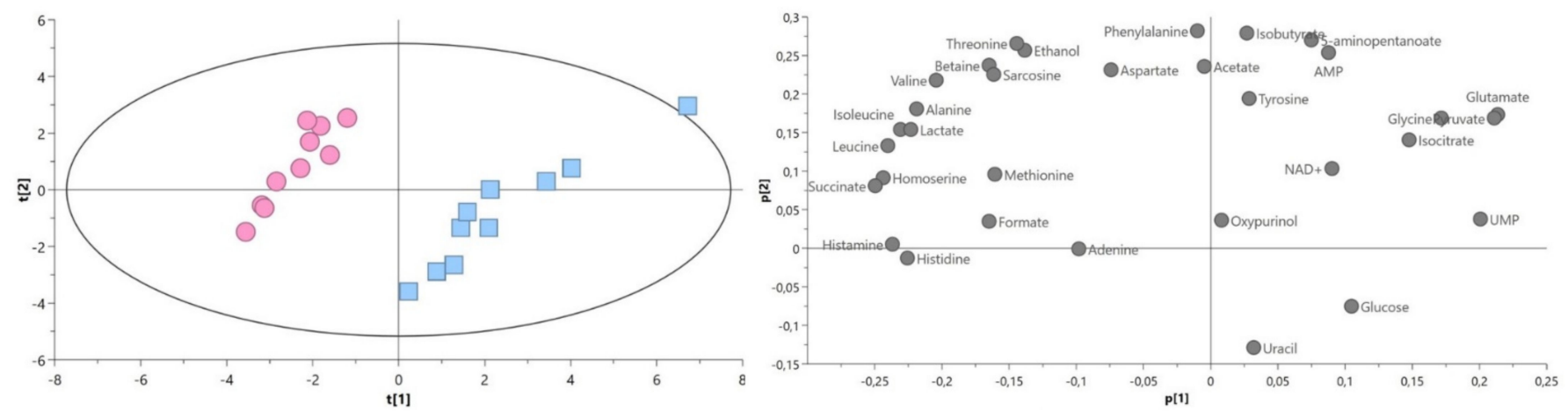

Figure 4. PCA model plot and corresponding loading plot of ${ }^{1} \mathrm{H}$ NMR data of intracellular metabolites of drug-resistant (blue squares) and drug-susceptible (red circle) P. aeruginosa strains. Symbols in the same color represent biological repetitions.

Supervised OPLS-DA analysis provides the strain's grouping. The CV-ANOVA test of this model gave statistically important results (model with parameters are available in Supplementary Materials (Figure S2)).

\subsubsection{Extracellular Metabolites}

For the PCA extracellular metabolites analysis, in addition to two types of bacterial strain isolates, a control group was also taken into consideration (the entire content of medium before bacterial cultivation).

The PCA score plot showed the difference between sample distributions for bacteria isolates with different antibiotic resistance, while the control group formed a separate 
data set. In a comparison of both P. aeruginosa strains with control, the first and second principal components (PC) accounted for $56.0 \%$ and $19.7 \%$ of the total variance in the data, respectively, $(\mathrm{R} 2 \mathrm{X}=0.996)$ (Figure $5 \mathrm{~A})$. The score plot showed the differences between the amino acids (glycine, alanine, tryptophan, leucine, lysine, methionine, threonine, and histidine), pyruvate, isobutyrate, acetate, tyrosine, and 6-hydroxynicotinate.
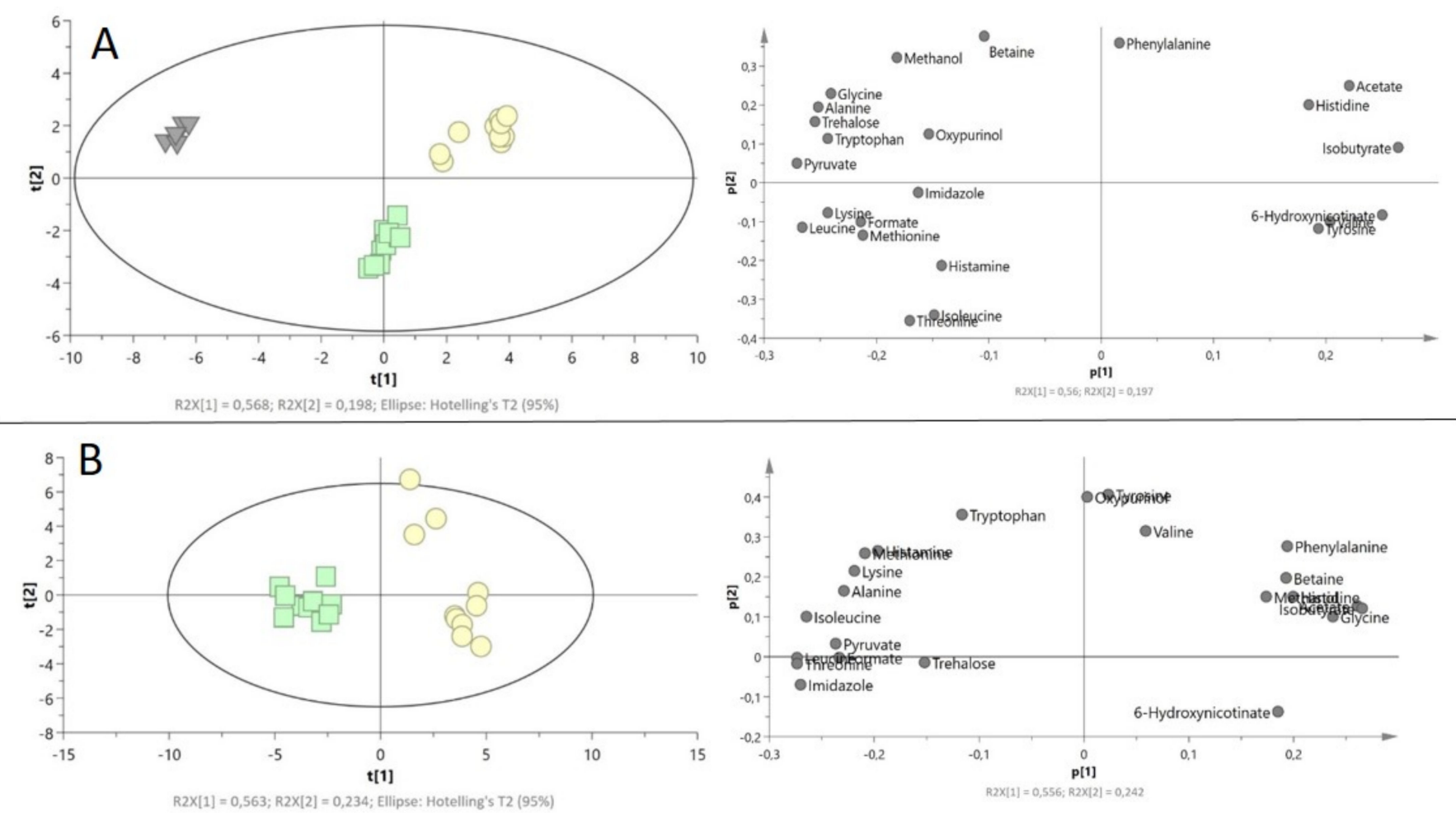

Figure 5. PCA model plots and corresponding loading plots for ${ }^{1} \mathrm{H}$ NMR data of extracellular metabolites of $P$. aeruginosa strains. (A) Both P. aeruginosa strains with control; (B) both P. aeruginosa strains. Drug-resistant extracellular (green squares), drug-susceptible extracellular (yellow circle), control—LB medium (gray triangles). Symbols in the same color represent biological repetitions.

The direct comparison between the P. aeruginosa strains showed a clear separation between the studied groups. The first and second principal components (PC) accounted, respectively, for $55.6 \%$ and $23.2 \%$ of the total variance. Differences were mainly observed in amino acid levels (Figure 5B).

For each comparison, the supervised OPLS-DA analysis provides the strain's grouping. The CV-ANOVA test of this model gave statistically important results (model with parameters are available in Supplementary Materials (Figure S3)). Information concerning an additional PCA single comparison of the drug-resistant $P$. aeruginosa strain with control and drug-susceptible P. aeruginosa strain with control is available in Supplementary Materials (Figure S4).

\subsection{Statistical Analysis}

\subsubsection{Intracellular Metabolites}

Among all the identified metabolites, 20 showed a statistically significant difference between susceptible and resistant strains $(p<0.05)$ (succinate, homoserine, histidine, histamine, lactate, alanine, glutamate, pyruvate, UMP, valine, isoleucine, leucine, betaine, methionine, formate, glycine, sarcosine, threonine, ethanol, and isocitrate).

In the same comparison, VIP scores greater than 1.00 were obtained for 16 overlapped metabolites (all statistically important metabolites, without glycine, threonine, succinate, and lactate). Detailed statistical data are shown in Table 2. 


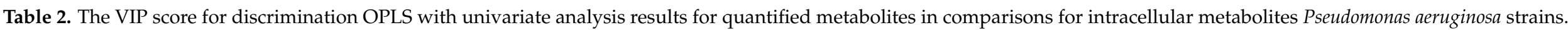

\begin{tabular}{|c|c|c|c|c|c|c|c|c|c|}
\hline $\begin{array}{l}\text { Compound } \\
\text { Group }\end{array}$ & Metabolite & $\begin{array}{l}\text { VIP Score for } \\
\text { OPLS-DA } \\
\text { Model }\end{array}$ & $\begin{array}{l}\text { Mean/Median * } \\
\text { Relative } \\
\text { Concentration R }\end{array}$ & $\begin{array}{l}\text { Mean/Median * } \\
\text { Relative } \\
\text { Concentration S }\end{array}$ & RSD R [\%] & RSD S [\%] & Fold Change R/S & $p$ Value & FDR $* *$ \\
\hline \multirow{11}{*}{ 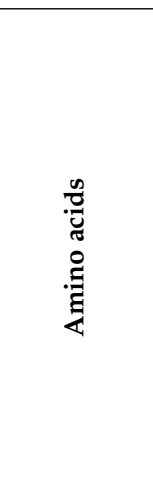 } & Histidine & 1.238 & 0.030 & 0.022 & 7.096 & 12.502 & 1.376 & $6.22 \times 10^{-7}$ & $6.63 \times 10^{-6}$ \\
\hline & Alanine & 1.282 & 0.206 & 0.152 & 11.021 & 8.278 & 1.358 & $3.23 \times 10^{-6}$ & $1.72 \times 10^{-5}$ \\
\hline & Glutamate & 1.247 & 0.308 & 0.371 & 10.271 & 5.964 & 0.830 & $6.48 \times 10^{-5}$ & $2.71 \times 10^{-4}$ \\
\hline & Valine & 1.246 & 0.185 & 0.140 & 13.759 & 9.833 & 1.323 & $1.06 \times 10^{-4}$ & $3.40 \times 10^{-4}$ \\
\hline & Isoleucine & 1.322 & $0.095^{\#}$ & $0.056^{\#}$ & 18.228 & 11.873 & 1.692 & $1.83 \times 10^{-4}$ & $4.87 \times 10^{-4}$ \\
\hline & Leucine & 1.359 & $0.383^{\#}$ & $0.203^{\#}$ & 18.323 & 8.907 & 1.887 & $1.83 \times 10^{-4}$ & $4.87 \times 10^{-4}$ \\
\hline & Glycine & 1.029 & 0.202 & 0.229 & 10.051 & 7.502 & 0.881 & $4.38 \times 10^{-3}$ & $8.76 \times 10^{-3}$ \\
\hline & Threonine & 1.034 & 0.042 & 0.038 & 10.142 & 5.496 & 1.109 & $1.62 \times 10^{-2}$ & $2.88 \times 10^{-2}$ \\
\hline & Aspartate & 0.707 & 0.068 & 0.062 & 15.953 & 14.564 & 1.093 & $2.11 \times 10^{-1}$ & $2.81 \times 10^{-1}$ \\
\hline & Phenylalanine & 0.705 & $0.113^{\#}$ & $0.121^{\#}$ & 14.269 & 7.127 & 0.933 & $5.21 \times 10^{-1}$ & $6.02 \times 10^{-1}$ \\
\hline & Tyrosine & 0.508 & 0.115 & 0.118 & 10.702 & 10.218 & 0.970 & $5.26 \times 10^{-1}$ & $6.02 \times 10^{-1}$ \\
\hline \multirow{4}{*}{ 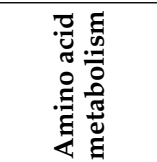 } & Homoserine & 1.358 & 0.619 & 0.392 & 9.718 & 5.487 & 1.578 & $1.85 \times 10^{-7}$ & $2.96 \times 10^{-6}$ \\
\hline & Histamine & 1.300 & 0.016 & 0.009 & 16.267 & 16.722 & 1.725 & $1.50 \times 10^{-6}$ & $1.13 \times 10^{-5}$ \\
\hline & Sarcosine & 1.048 & 0.022 & 0.016 & 19.383 & 28.508 & 1.386 & $5.94 \times 10^{-3}$ & $1.12 \times 10^{-2}$ \\
\hline & 5-aminopentanoate & 0.786 & 0.266 & 0.279 & 11.883 & 8.474 & 0.954 & $3.22 \times 10^{-1}$ & $3.96 \times 10^{-1}$ \\
\hline \multirow{3}{*}{ 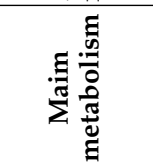 } & Pyruvate & 1.231 & 0.199 & 0.238 & 10.392 & 5.611 & 0.834 & $7.63 \times 10^{-5}$ & $2.71 \times 10^{-4}$ \\
\hline & Isocitrate & 0.885 & 0.169 & 0.193 & 16.536 & 3.586 & 0.873 & $2.18 \times 10^{-2}$ & $3.51 \times 10^{-2}$ \\
\hline & Glucose & 0.602 & 0.031 & 0.036 & 25.176 & 14.910 & 0.864 & $1.22 \times 10^{-1}$ & $1.77 \times 10^{-1}$ \\
\hline Cofactor & NAD+ & 0.559 & 0.031 & 0.034 & 7.776 & 11.658 & 0.938 & $1.71 \times 10^{-1}$ & $2.38 \times 10^{-1}$ \\
\hline \multirow{4}{*}{ 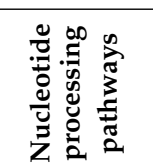 } & UMP & 1.104 & 0.009 & 0.012 & 13.476 & 6.289 & 0.799 & $7.52 \times 10^{-5}$ & $2.71 \times 10^{-4}$ \\
\hline & Adenine & 0.54 & $0.011^{\#}$ & $0.010^{\#}$ & 8.239 & 13.275 & 1.103 & $1.21 \times 10^{-1}$ & $1.77 \times 10^{-1}$ \\
\hline & AMP & 0.797 & 0.067 & 0.072 & 12.569 & 12.192 & 0.932 & $2.21 \times 10^{-1}$ & $2.83 \times 10^{-1}$ \\
\hline & Uracil & 0.364 & 0.010 & 0.010 & 26.399 & 19.769 & 0.964 & $7.31 \times 10^{-1}$ & $8.06 \times 10^{-2}$ \\
\hline \multirow{7}{*}{ 离 } & Lactate & 1.284 & 0.274 & 0.186 & 12.175 & 11.863 & 1.471 & $1.76 \times 10^{-6}$ & $1.13 \times 10^{-5}$ \\
\hline & Betaine & 1.083 & 8.428 & 7.200 & 11.685 & 7.209 & 1.171 & $2.63 \times 10^{-3}$ & $6.47 \times 10^{-3}$ \\
\hline & Formate & 0.908 & 0.009 & 0.007 & 13.181 & 16.178 & 1.237 & $4.33 \times 10^{-3}$ & $8.76 \times 10^{-3}$ \\
\hline & Ethanol & 0.991 & 0.075 & 0.065 & 14.898 & 6.110 & 1.153 & $2.19 \times 10^{-2}$ & $3.51 \times 10^{-2}$ \\
\hline & Isobutyrate & 0.711 & $0.018^{\#}$ & $0.017^{\#}$ & 19.107 & 12.686 & 1.078 & $8.50 \times 10^{-1}$ & $9.07 \times 10^{-1}$ \\
\hline & Oxypurinol & 0.1 & $0.034^{\#}$ & $0.034^{\#}$ & 9.793 & 9.363 & 0.997 & $9.10 \times 10^{-1}$ & $9.39 \times 10^{-1}$ \\
\hline & Acetate & 0.59 & 0.066 & 0.065 & 13.588 & 25.511 & 1.006 & $9.48 \times 10^{-1}$ & $9.48 \times 10^{-1}$ \\
\hline
\end{tabular}

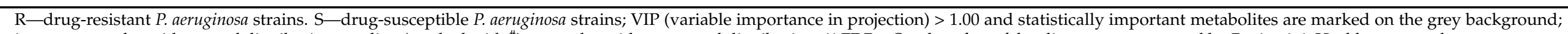

${ }^{*}$ mean-samples with normal distribution; median (marked with ${ }^{*}$ —-samples without normal distribution; ${ }^{* *}$ FDR—Q values from false discovery rate control by Benjamini-Hochberg procedure. 
Among the differentiating metabolites the relative concentration of succinate, homoserine, lactate, alanine, valine, isoleucine, leucine, betaine, histidine, histamine, methionine, formate, sarcosine, threonine, and ethanol were upregulated in the group of drug-resistant $P$. aeruginosa, while only five metabolites were at the higher-level for drug-sensitive samplesglutamate, pyruvate, UMP, glycine, isocitrate (Figure 6).
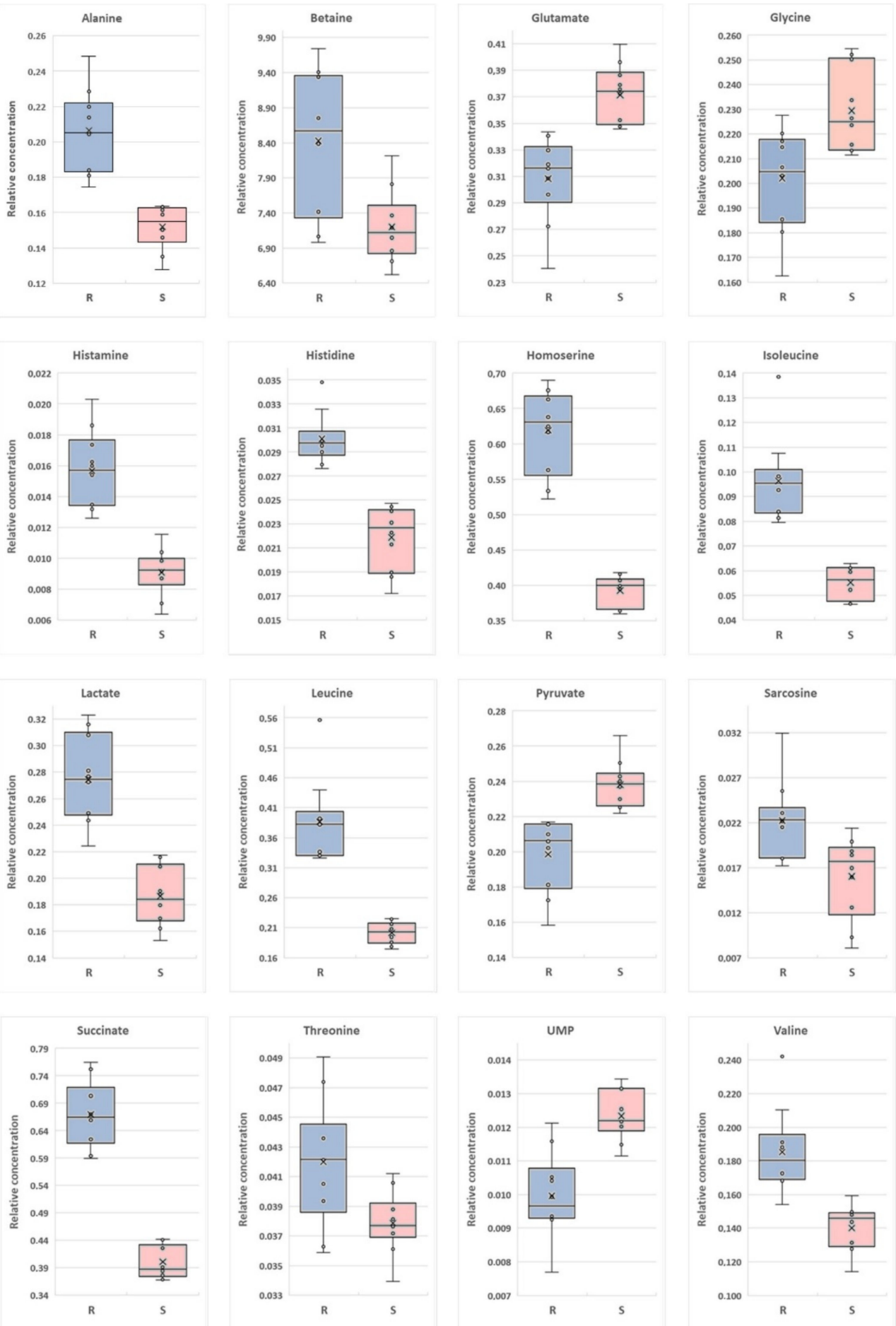

Figure 6. Boxplots for intracellular metabolites with VIP (Variable Importance in Projection) scores above 1.00 are statistically important after $p$-value adjustment $(q<0.05)$. Red bars-S-drugsusceptible strain; blue bars- $\mathrm{R}-$ drug-resistant strain. 


\subsubsection{Extracellular Metabolites}

The comparison of the drug-resistant $P$. aeruginosa strain with the drug-susceptible strain enabled the identification of 16 statistically significant metabolites $(p<0.05)$ (acetate, alanine, betaine, glycine, formate, histidine, imidazole, isobutyrate, isoleucine, leucine, lysine, methanol, phenylalanine, pyruvate, threonine and 6-hydroxynicotinate), while a VIP scores greater than 1.00 were found for nine metabolites (acetate, glycine, imidazole, isobutyrate, isoleucine, leucine, phenylalanine, pyruvate, and threonine).

The comparison of the drug-resistant P. aeruginosa strain with the control (LB medium) enabled the identification of 17 statistically significant metabolites $(p<0.05)$ (acetate, alanine, betaine, glycine, isobutyrate, leucine, lysine, methanol, methionine, phenylalanine, pyruvate, trehalose, threonine, tryptophan, tyrosine, valine and 6-hydroxynicotinate). In this case, VIP scores greater than 1.00 were obtained for 15 metabolites (all statistically important metabolites, without acetate, and threonine).

The comparison of the drug-susceptible $P$. aeruginosa strain with the control (LB medium) identified 19 statistically significant metabolites $(p<0.05)$ (acetate, alanine, betaine, glycine, histidine, isobutyrate, isoleucine, leucine, lysine, methanol, methionine, phenylalanine, pyruvate, trehalose, threonine, tryptophan, tyrosine, valine and 6hydroxynicotinate). 14 metabolites had VIP score greater than 1.00 (all statistically significant metabolites, without isoleucine, histidine, methionine, imidazole, and betaine). Detailed statistical data are available in Table 3.

Among the differentiating metabolites, the relative concentration of alanine, formate, imidazole, isoleucine, leucine, lysine, pyruvate, and threonine was upregulated in the group of drug-resistant $P$. aeruginosa vs. drug-susceptible strains. Eight metabolites were at a higher level in drug-susceptible samples-6-hydroksynicotinate, acetate, betaine, glycine, histidine, isobutyrate, methanol, and phenylalanine. The relative concentration of three metabolites was lower in bacterial culture media than the control medium-glycine, leucine, and pyruvate. The concentrations of isobutyrate and acetate were higher in cell culture media than in control samples (Figure 7).

\subsection{Bioinformatics Analysis}

Bioinformatics analysis was used to produce a graphical representation of compounds, enzymes, reactions, modules, and pathways with information on how the input metabolites found in the statistical analysis reach the suggested pathways and on how these pathways cross-talk. An interactive version of the graphic for analysis is also available. For this analysis metabolites with statistical importance and VIPs greater than 1.00 were chosen.

\subsubsection{Intracellular Metabolites}

The graphical representation of compounds, enzymes, reactions, modules, pathways, with information on how the input metabolites reach the suggested pathways, and on how these pathways cross-talk for intracellular metabolites is shown in Figure 8.

The main pathways connected with these compounds are ABC transporters, vancomycin metabolism pathway, and amino acids pathways, especially glycine, serine, threonine, alanine, and pyruvate metabolism. 


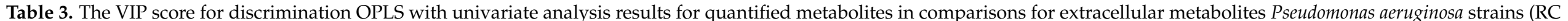
-relative concentration).

\begin{tabular}{|c|c|c|c|c|c|c|c|c|c|c|c|c|c|c|c|c|c|c|c|}
\hline \multirow[b]{2}{*}{ Group } & \multirow[b]{2}{*}{ Metabolites } & \multirow[b]{2}{*}{$\begin{array}{c}\text { Mean/ } \\
\text { Median RC } \\
\mathbf{R}^{*}\end{array}$} & \multirow[b]{2}{*}{$\begin{array}{l}\text { Mean/ } \\
\text { Median RC } \\
\text { s* }^{*}\end{array}$} & \multirow[b]{2}{*}{$\begin{array}{c}\text { Mean/ } \\
\text { Mediaan RC } \\
\text { C }^{*}\end{array}$} & \multirow[b]{2}{*}{$\underset{[\%]}{\text { RSD R }}$} & \multirow[b]{2}{*}{$\underset{[\%]}{\mathrm{RSD} R}$} & \multirow[b]{2}{*}{$\underset{[\%]}{\text { RSD K }}$} & \multicolumn{4}{|c|}{ R vs. $\mathrm{S}$} & \multicolumn{4}{|c|}{ Cvs. R } & \multicolumn{4}{|c|}{ Cvs. S } \\
\hline & & & & & & & & $\begin{array}{l}\text { VIP } \\
\text { Score }\end{array}$ & $\begin{array}{l}\text { Fold } \\
\text { Change } \\
\text { R/S }\end{array}$ & $p$ Value & $\underset{* * *}{\mathrm{FDR}}$ & $\begin{array}{l}\text { VIP } \\
\text { Score }\end{array}$ & $\begin{array}{l}\text { Fold } \\
\text { Change } \\
\text { C/R }\end{array}$ & $p$ Value & FDR & $\begin{array}{l}\text { VIP } \\
\text { Score }\end{array}$ & $\begin{array}{l}\text { Fold } \\
\text { Change } \\
\text { C/S }\end{array}$ & $p$ Value & FDR \\
\hline \multirow{11}{*}{ 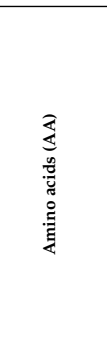 } & Threonine & 6.583 & 4.868 & 6.128 & 3.623 & 5.620 & 1.900 & 1.197 & 1.352 & $1.37 \times 10^{-11}$ & $8.24 \times 10^{-11}$ & 0.886 & 0.931 & $1.57 \times 10^{-3}$ & $2.22 \times 10^{-3}$ & 1.083 & 1.259 & $2.49 \times 10^{-7}$ & $7.46 \times 10^{-7}$ \\
\hline & Leucine & 8.607 & 6.050 & 10.65 & 2.352 & 8.221 & 5.513 & 1.201 & 1.423 & $4.14 \times 10^{-9}$ & $1.99 \times 10^{-8}$ & 1.133 & 1.237 & $1.03 \times 10^{-3}$ & $1.54 \times 10^{-3}$ & 1.118 & 1.76 & $6.48 \times 10^{-80}$ & $2.22 \times 10^{-9}$ \\
\hline & Isoleucine & 2.676 & 2.191 & 2.516 & 4.134 & 8.724 & 8.159 & 1.115 & 1.221 & $1.75 \times 10^{-6}$ & $6.00 \times 10^{-6}$ & 0.584 & 0.94 & $6.76 \times 10^{-2}$ & $8.54 \times 10^{-2}$ & 0.881 & 1.148 & $9.65 \times 10^{-3}$ & $1.22 \times 10^{-2}$ \\
\hline & Glycine & 0.093 & 0.138 & 1.155 & 16.681 & 11.456 & 1.660 & 1.080 & 0.678 & $5.93 \times 10^{-6}$ & $1.78 \times 10^{-5}$ & 1.203 & 12.363 & $5.73 \times 10^{-21}$ & $4.93 \times 10^{-20}$ & 1.14 & 8.387 & $1.10 \times 10^{-20}$ & $1.78 \times 10^{-19}$ \\
\hline & Phenylalanine & 2.015 & 2.407 & 2.352 & 2.981 & 8.182 & 2.468 & 1.078 & 0.837 & $9.95 \times 10^{-5}$ & $2.39 \times 10^{-4}$ & 1.146 & 1.167 & $1.22 \times 10^{-7}$ & $2.94 \times 10^{-7}$ & 0.696 & 0.977 & $4.31 \times 10^{-1}$ & $4.50 \times 10^{-1}$ \\
\hline & $\begin{array}{l}\text { Histidine } \\
\end{array}$ & 0.155 & 0.195 & 0.142 & 14.571 & 11.601 & 4.770 & 0.944 & 0.796 & $9.54 \times 10^{-4}$ & $1.76 \times 10^{-3}$ & 0.376 & 0.916 & $1.19 \times 10^{-1}$ & $1.36 \times 10^{-1}$ & 0.947 & 0.729 & $2.15 \times 10^{-5}$ & $4.68 \times 10^{-5}$ \\
\hline & Alanine & 1.993 & 1.835 & 6.954 & 3.342 & 5.546 & 1.453 & 0.974 & 1.086 & $2.20 \times 10^{-3}$ & $3.78 \times 10^{-3}$ & 1.204 & 3.489 & $6.17 \times 10^{-21}$ & $4.93 \times 10^{-20}$ & 1.139 & 3.79 & $6.66 \times 10^{-4}$ & $9.40 \times 10^{-4}$ \\
\hline & Lysine & 10.993 & 10.17 & 11.918 & 2.894 & 6.608 & 1.520 & 0.949 & 1.081 & $4.00 \times 10^{-3}$ & $6.00 \times 10^{-3}$ & 1.042 & 1.084 & $4.71 \times 10^{-5}$ & $8.69 \times 10^{-5}$ & 1.026 & 1.172 & $8.28 \times 10^{-6}$ & $1.99 \times 10^{-5}$ \\
\hline & Valine & 3.985 & $4.177^{\#}$ & 3.493 & 3.649 & 5.396 & 4.115 & 0.752 & 0.954 & $8.90 \times 10^{-2}$ & $1.19 \times 10^{-1}$ & 1.038 & 0.877 & $3.23 \times 10^{-5}$ & $6.45 \times 10^{-5}$ & 1.031 & 0.836 & $6.66 \times 10^{-4}$ & $9.40 \times 10^{-4}$ \\
\hline & Methionine & 0.949 & $0.202 \#$ & 1.205 & 5.045 & 97.014 & 1.313 & 0.943 & 4.706 & $1.40 \times 10^{-1}$ & $1.61 \times 10^{-1}$ & 1.154 & 1.27 & $2.82 \times 10^{-9}$ & $9.66 \times 10^{-9}$ & 0.943 & 5.977 & $1.27 \times 10^{-2}$ & $1.52 \times 10^{-2}$ \\
\hline & Tyrosine & 0.861 & $0.861^{\#}$ & 0.599 & 4.827 & 15.001 & 0.640 & 0.817 & 1.000 & $4.73 \times 10^{-1}$ & $4.73 \times 10^{-1}$ & 1.160 & 0.696 & $6.50 \times 10^{-9}$ & $1.73 \times 10^{-8}$ & 1.022 & 0.696 & $6.66 \times 10^{-4}$ & $9.40 \times 10^{-4}$ \\
\hline $\begin{array}{c}\text { AA } \\
\text { metabolism }\end{array}$ & Histamine & 0.283 & 0.167 \# & 0.273 & 4.487 & 38.007 & 2.369 & 0.900 & 1.700 & $1.40 \times 10^{-1}$ & $1.61 \times 10^{-1}$ & 0.518 & 0.965 & $1.30 \times 10^{-1}$ & $1.42 \times 10^{-1}$ & 0.809 & 1.641 & $2.54 \times 10^{-1}$ & $2.78 \times 10^{-1}$ \\
\hline \multirow{10}{*}{ 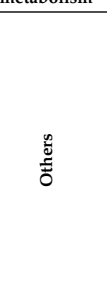 } & Imidazole & 0.078 & 0.047 & $0.055^{\#}$ & 3.930 & 5.241 & 72.63 & 1.221 & 1.672 & $1.63 \times 10^{-15}$ & $3.91 \times 10^{-14}$ & 0.463 & 0.712 & $5.94 \times 10^{-1}$ & $5.94 \times 10^{-1}$ & 0.681 & 1.19 & $6.66 \times 10^{-4}$ & $9.40 \times 10^{-4}$ \\
\hline & Oxypurinol & 0.096 & 0.100 & $0.22{ }^{\#}$ & 3.293 & 11.458 & 52.044 & 0.768 & 0.961 & $3.22 \times 10^{-1}$ & $3.52 \times 10^{-1}$ & 0.690 & 2.347 & $5.94 \times 10^{-1}$ & $5.94 \times 10^{-1}$ & 0.662 & 2.255 & $5.94 \times 10^{-1}$ & $5.94 \times 10^{-1}$ \\
\hline & Isobutyrate & 0.436 & 0.692 & 0.204 & 6.416 & 3.999 & 19.06 & 1.221 & 0.630 & $5.94 \times 10^{-14}$ & $7.12 \times 10^{-13}$ & 1.164 & 0.468 & $5.73 \times 10^{-9}$ & $1.72 \times 10^{-8}$ & 1.134 & 0.295 & $4.70 \times 10^{-13}$ & $2.82 \times 10^{-12}$ \\
\hline & Acetate & 3.486 & 10.284 & 2.589 & 32.191 & 7.080 & 2.005 & 1.200 & 0.339 & $4.04 \times 10^{-12}$ & $3.23 \times 10^{-11}$ & 0.522 & 0.743 & $3.24 \times 10^{-2}$ & $4.32 \times 10^{-2}$ & 1.135 & 0.252 & $6.94 \times 10^{-11}$ & $2.77 \times 10^{-10}$ \\
\hline & Pyruvate & 0.184 & 0.153 & 0.256 & 5.221 & 5.938 & 1.400 & 1.131 & 1.203 & $7.16 \times 10^{-7}$ & $2.86 \times 10^{-6}$ & 1.178 & 1.388 & $7.05 \times 10^{-10}$ & $2.82 \times 10^{-9}$ & 1.129 & 1.669 & $3.91 \times 10^{-12}$ & $1.88 \times 10^{-11}$ \\
\hline & Betaine & 5.167 & 5.667 & 6.108 & 4.576 & 4.925 & 1.544 & 0.970 & 0.912 & $4.17 \times 10^{-4}$ & $9.10 \times 10^{-4}$ & 1.111 & 1.182 & $1.26 \times 10^{-6}$ & $2.74 \times 10^{-6}$ & 0.818 & 1.078 & $4.91 \times 10^{-3}$ & $6.55 \times 10^{-3}$ \\
\hline & Formate & $\begin{array}{ll}0.048 \\
\end{array}$ & 0.017 & $0.092 \#$ & 40.445 & 24.975 & 49.725 & 0.989 & 2.816 & $6.25 \times 10^{-4}$ & $1.25 \times 10^{-3}$ & 0.58 & 1.924 & $7.53 \times 10^{-2}$ & $9.03 \times 10^{-2}$ & 0.94 & 5.419 & $7.53 \times 10^{-2}$ & $8.60 \times 10^{-2}$ \\
\hline & Trehalose & $0.121^{\#}$ & 0.084 & 0.317 & 27.213 & 7.319 & 3.308 & 0.744 & 1.444 & $3.85 \times 10^{-1}$ & $4.01 \times 10^{-1}$ & 1.174 & 2.613 & $6.66 \times 10^{-4}$ & $1.07 \times 10^{-3}$ & 1.137 & 3.773 & $8.92 \times 10^{-17}$ & $7.14 \times 10^{-16}$ \\
\hline & Methanol & 0.035 & 0.043 & 0.062 & 6.492 & 13.301 & 2.112 & 0.859 & 0.826 & $2.45 \times 10^{-3}$ & $3.92 \times 10^{-3}$ & 1.193 & 1.741 & $5.12 \times 10^{-12}$ & $2.46 \times 10^{-11}$ & 1.03 & 1.439 & $9.90 \times 10^{-7}$ & $2.64 \times 10^{-6}$ \\
\hline & 6-Hydroxynicotinate & 0.113 & 0.126 & $0.054 \#$ & 3.153 & 10.280 & 35.106 & 0.813 & 0.899 & $1.28 \times 10^{-2}$ & $1.80 \times 10^{-2}$ & 1.078 & 0.478 & $6.66 \times 10^{-4}$ & $1.07 \times 10^{-8-3}$ & 1.038 & 0.43 & $6.66 \times 10^{-4}$ & $9.40 \times 10^{-4}$ \\
\hline
\end{tabular}

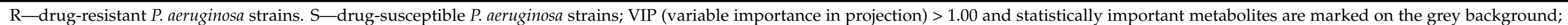

${ }^{*}$ mean—samples with normal distribution; median (marked with ${ }^{*}$ —-samples without normal distribution; ${ }^{* *}$ FDR—Q values from false discovery rate control by Benjamini-Hochberg procedure. 

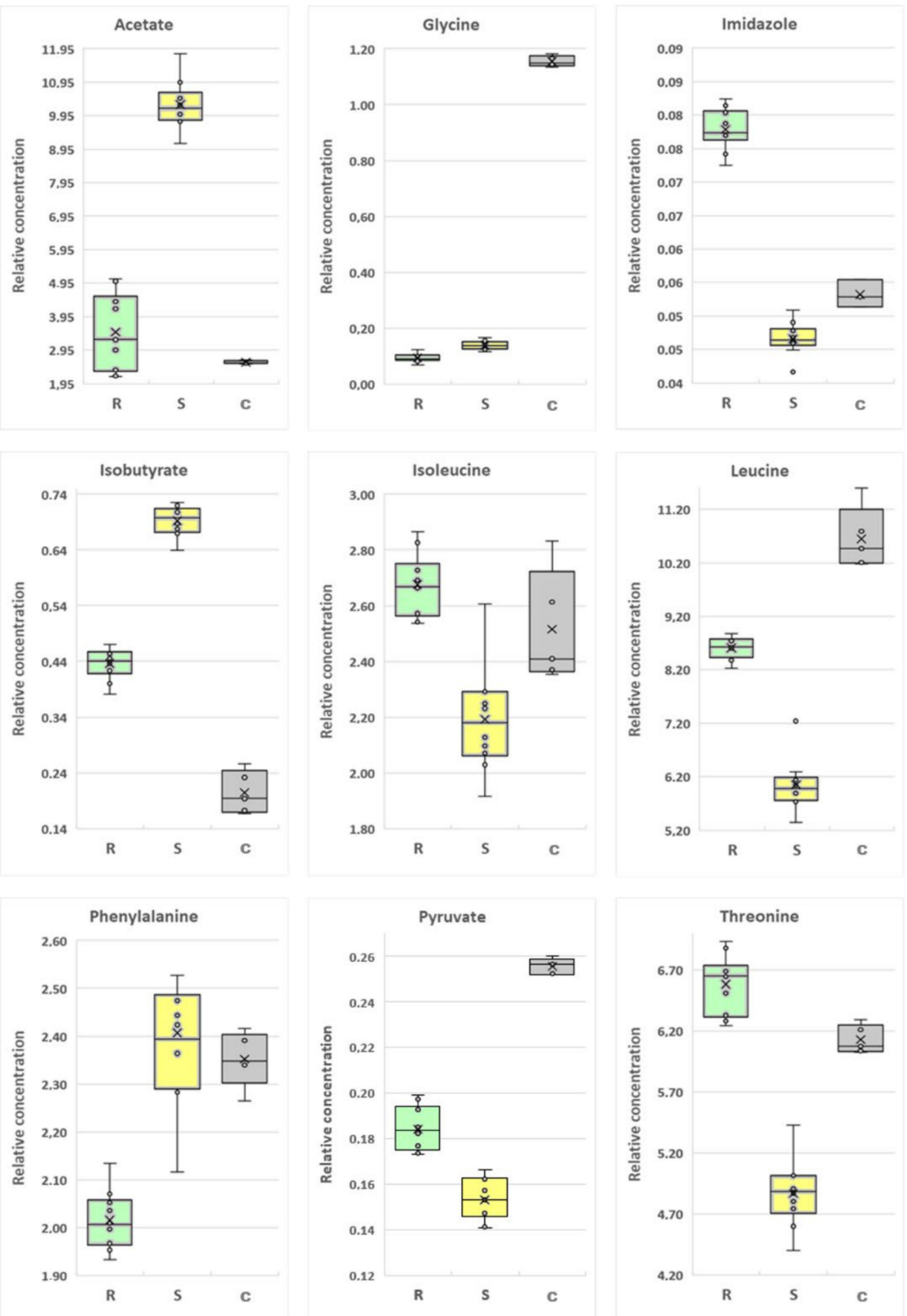

Figure 7. Boxplots for extracellular metabolites with VIP (Variable Importance in Projection) scores above 1.00 and statistically important after $p$-value adjustment $(q<0.05)$. Yellow bars-S-drugsusceptible strain. green bars $-\mathrm{R}-\mathrm{drug}$-resistant strain, gray bars $-\mathrm{C}-$ control (medium LB). 


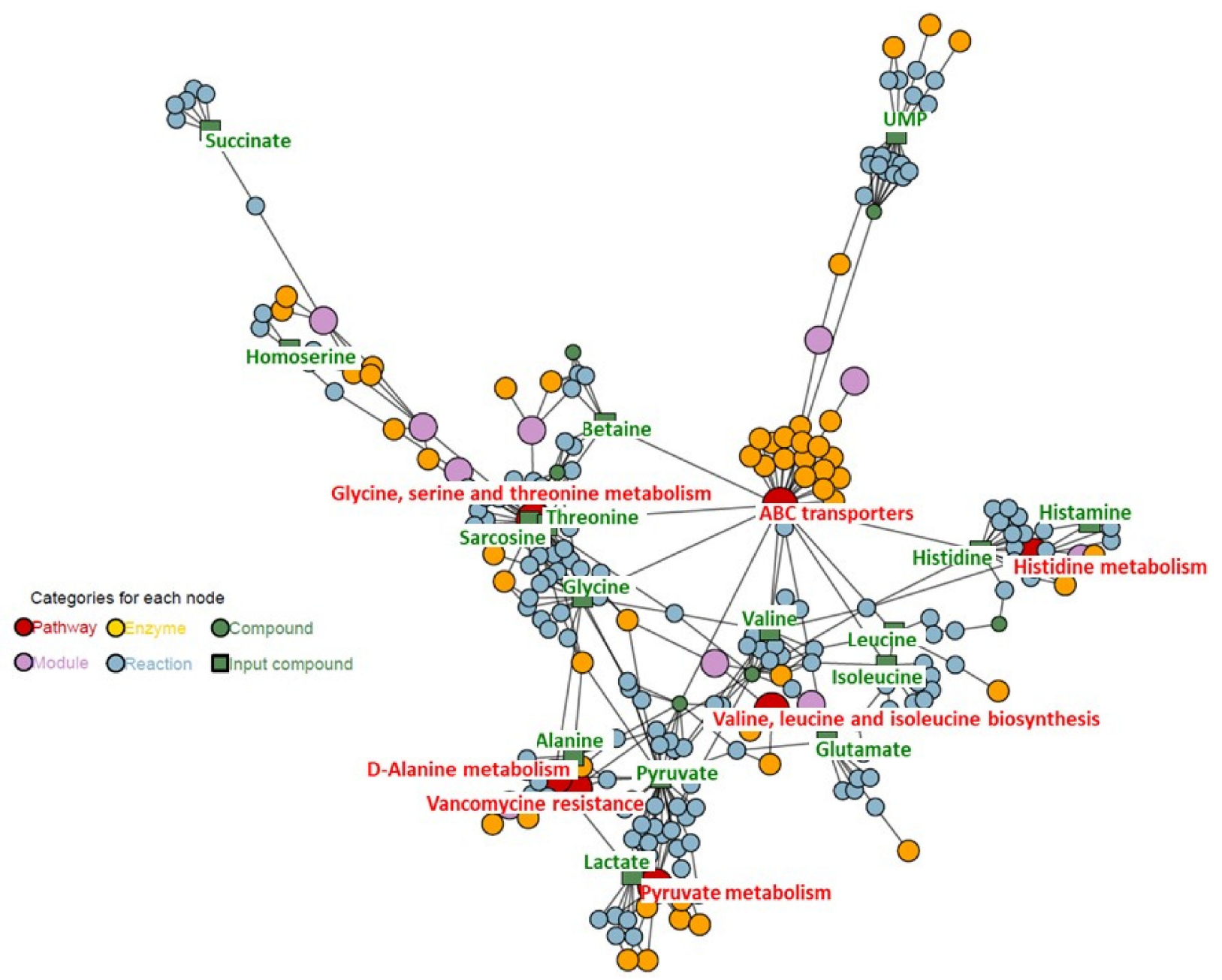

Figure 8. Results of the node prioritization by FELLA in the Pseudomonas aeruginosa strain (intracellular analysis).

\subsubsection{Extracellular Metabolites}

The graphical representation of compounds, enzymes, reactions, modules, pathways, and information on how the input metabolites reach the suggested pathways, and on how these pathways cross-talk for extracellular metabolites is shown in Figure 9.

The main pathways connected with these compounds are ABC transporters, vancomycin metabolism pathway, and amino acids pathways.

The interactive versions of Figures 8 and 9 are available in Supplementary Materials (Files S1 and S2). 


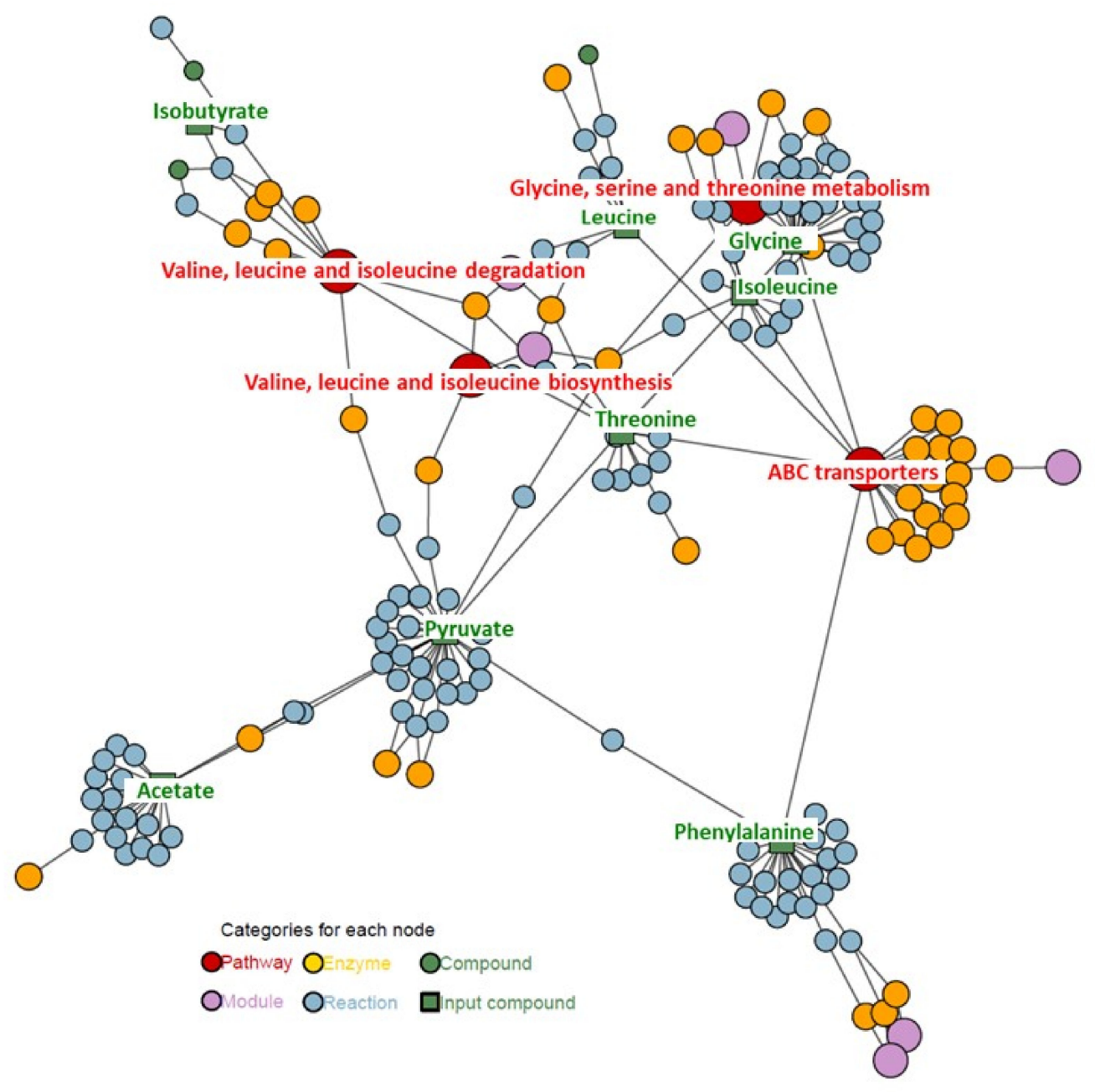

Figure 9. Results of the node prioritization by FELLA in the Pseudomonas aeruginosa strain (extracellular analysis).

\section{Discussion}

Increasing antibiotic resistance leads to the development of alternative chemotherapeutics dedicated to treating bacterial infections. In the case of $P$. aeruginosa infections, treatment with $\beta$-lactams, fluoroquinolones, and aminoglycosides is widely used. In multidrug resistant strains, ceftolozan/tazobactam and colistin could also be administered, but these can cause toxic and adverse side effects $[3,4,12]$. New therapeutic strategies may involve the addition of compounds improving the activity of traditional antibiotics [27]. Understanding the exact mechanisms of action of currently used drugs could support this process, which is why it is so important to perform analyses at various molecular levels [8]. Insights about differences between drug-resistant and drug-sensitive strains can be given by metabolomics studies, where the differences in the low-molecular-weight compounds regulation reflect the changes in biochemical pathways [28]. This approach may be helpful for the delineation of molecular targets and drug design [29]. In addition, bacteriostatic compounds based on new structures and mechanisms of action are being designed [30]. Information regarding the prevalence of antibiotic resistance mechanisms would support the development process.

However, these strategies will probably not be universal. In such a situation, selection of effective therapy should be based on a detailed analysis of resistance mechanisms present in an individual clinical strain. Treatment of antibiotic-resistant bacteria is likely to require an individual approach. Therefore, the development of a fast and accurate diagnostic tool 
seems to be necessary. Our results show that the metabolomics approach with the use of ${ }^{1} \mathrm{H}$ NMR spectroscopy may establish new horizons in this area [29].

The analysis of all accessible sources of data may provide an interesting insights into the evolutionary adaptation of $P$. aeruginosa. Among intracellular metabolites there is an interesting relationship between pyruvate and its reduction product-lactate. In the antibiotic resistant strain, the level of pyruvate was decreased while the level of lactate is increased, in comparison to antibiotic-susceptible strain. This may be the result of a more intense reduction reaction of pyruvate in the drug-resistant strain since none of the strains secreted these compounds to the culture medium. However, research in cystic fibrosis (CF) patients' samples shows that lactate is a major component in sputum, and could be an important infection factor [31]. Pyruvate seems also to be associated with BCAA biosynthesis (valine, leucine, and isoleucine), which were upregulated in the drugresistant strain [32]. Moreover, significant differences were observed in the intracellular concentrations of isocitrate (lower in the resistant strain) and succinate (higher in the resistant strain).

In the majority of cases, the identified intracellular amino acids were at the higher level in the case of the antibiotic-resistant strain. The exceptions were glycine, glutamate, and tyrosine whose concentrations were significantly higher in the antibiotic-resistant strain. According to literature data, the amino acid conversion pathways are more efficient in antibiotic-resistant strains [33]. Our results have shown clearly changes in amino acids in both strains. Furthermore, the metabolic modification to amino acids as carbon sources is essential in antibiotic resistance $[14,34,35]$.

In the antibiotic resistance strain, the relative concentration of UMP was lower. The synthesis of UMP has been shown to play an important role in sustaining virulence, biofilm formation, and antibiotic resistance in P. aeruginosa [14,27]. The production of biofilm in bacteria creates a physical barrier and reduces the effectiveness of treatment with various antibiotics. Furthermore, biofilm-formation is a critical mechanism of adaptive resistance [2]. Our results seem to confirm this observation.

Analysis of culture media revealed that the tested strains differed in amino acid turnover patterns. Both strains used all available glutamate, aspartate, asparagine, and pyroglutamate. However, for the susceptible strain, some of metabolites were taken from the medium at a higher level: alanine, leucine, lysine, and methionine, while threonine, histamine, and isoleucine were mostly utilized only by the antibiotic susceptible strain. The common metabolite for both types of isolates is glycine which was drained from the cultivation medium and seems to be one of the crucial nutrients. Different amino acid utilization patterns may result from reduced expression of transport proteins. The FELLA analysis results suggest that amino acid uptake may be interrupted. The KEGG database includes only information regarding amino acid ABC-transporters localized in the inner membrane [36]. These proteins are not considered antibiotic transporters; however, their activity depends on the presence of porins transporting amino acids from the environment into the periplasmatic space (these relations are not included in KEGG). Porins (such as OprD) are recognized as structures necessary for antibiotic uptake. Our results suggest that resistance of a strain to some $\beta$-lactams may depend on the reduced expression of porin, also resulting in reduced amino acid uptake. Elevated glycine uptake in the case of the antibiotic-resistant strain may result from a lack of threonine absorption. To satisfy the cell threonine demand, the antibiotic-resistant strain probably synthesizes this amino acid from glycine [35,37]. Moreover, in P. aeruginosa enzymes required for sarcosine synthesis from glycine were identified [38]. The different amino acid metabolism could originate for three reasons: different protein turnover, influx-outflux equilibrium, or bacteria amino acid biosynthesis. This last phenomenon can be caused by all the bacterial organism biochemical machinery for proteinogenic amino acid synthesis [33,37].

In the culture medium, acetate and isobutyrate were also present. The concentration of both compounds was higher in the case of the antibiotic-susceptible strain. These compounds are products of the catabolic metabolism of amino acids. Increased concentration 
of acetate and isobutyrate may indicate more intense metabolic activity of the antibiotic susceptible strain. The concentration of acetate in the cultivation medium showed a negative correlation with infection length in CF patients [34]. Reduced production of acetate is considered a sign of adaptation of a pathogen to the environment in the respiratory tracts of CF patients.

Observed metabolic differences between examined strains may result from starvation response (SR) activation in the drug-resistant strain. SR relies on the presence of the ppGpp molecule produced by two enzymes relA and spoT. RelA becomes active when the cell suffers amino acid limitation. SpoT produces ppGpp in response to sugar, iron, and fatty acid deficiency. Both proteins were identified in P. aeruginosa [15]. SR activation influences gene expression involved in glycolysis, the TCA cycle, and the amino acid synthesis pathway. In E. coli, expression of the majority of enzymes for amino acid synthesis is upregulated [25]. Moreover, the expression of glyoxalate-producing enzymes is also increased. On the other hand, expression of enzymes producing oxalacetate from succinate is reduced. If SR mechanisms in P. aeruginosa cause a transcriptional response similar to the one observed in E. coli, altered enzyme expression may explain the observed changes in intracellular metabolite concentration.

\section{Conclusions}

The treatment of bacterial infections is a significant problem, especially when in pathogens form biofilm structures. Despite novel pharmacotherapy restoring CFTR functionality being available, CF patients still suffer from bacterial infections [39]. Therefore, research focused on the treatment of bacterial lung infections is still needed.

Our results show that the metabolic differences between antibiotic-resistant and antibiotic-susceptible bacteria strains may be linked with the activity of antibiotic resistance mechanisms. Comparison of intracellular and extracellular metabolite profiles showed differences between drug-resistant and drug-susceptible P. aeruginosa strains within the intracellular amino acid pool. The intracellular free amino acid concentration results from the balance between different processes: protein synthesis, uptake from the environment, and their biosynthesis and degradation. This information may be helpful in the selection of the most effective therapy and targets for future drugs.

\section{Materials and Methods}

\subsection{Bacterial Strains and Culture Conditions}

In this study, two P. aeruginosa strains were analyzed: PAW17 (antibiotic susceptible) and PAW23 (antibiotic resistant). The strains were isolated from patients suffering from CF in the Mother and Child Institute in Warsaw. For long-term storage, strains were kept as glycerol preserved suspensions at $-80^{\circ} \mathrm{C}$.

After thawing from $-80^{\circ} \mathrm{C}$, the bacteria were grown on Miller's LB Broth agar (BioShop) overnight at $37^{\circ} \mathrm{C}$. In the next step, pre-culture was prepared. $5 \mathrm{~mL}$ of liquid LB medium in a test tube was inoculated with a single colony from the agar plate and incubated for $24 \mathrm{~h}$ at $37^{\circ} \mathrm{C}$ with shaking (180 r.p.m.). After that, $100 \mathrm{~mL}$ of the culture in a $300 \mathrm{~mL}$ conical flask was prepared (initial $\mathrm{OD}_{600 \mathrm{~nm}}=0.1$ ) and incubated for $24 \mathrm{~h}$ under the same conditions. The strains' breeding for metabolomics analysis was performed without antibiotic treatment. After $24 \mathrm{~h}$ of cultivation, both strains were in the stationary phase.

To collect bacterial cells the culture was centrifuged (19,000 r.c.f., $5 \mathrm{~min}, 4^{\circ} \mathrm{C}$ ) (sigma 3-18 KS, Polygen), and the bacterial pellet was washed with $0.9 \% \mathrm{NaCl}$ solution. Culture medium samples were stored at $-80^{\circ} \mathrm{C}$. The bacterial pellets were lyophilized (ScanvacCoolsave, Labogene) and stored at $-80^{\circ} \mathrm{C}$. Before extraction, each sample was weighted in tubes (Eppendorf). The entire protocol was repeated for each strain in ten biological repetitions. Additionally, to compare the levels of extracellular metabolites with fresh medium, five technical repetitions of fresh LB medium samples were analyzed. 


\subsection{Antibiotic Resistance}

The susceptibility of P. aeruginosa strains to most antibiotics was determined by the disc diffusion method. The bacterial suspension with a density equal to $0.5 \mathrm{McF}$ arland was inoculated with a swab on Mueller-Hinton II Agar. The following antibiotic discs were placed on the seeded medium: amikacin $(30 \mu \mathrm{g})$, netilmicin $(10 \mu \mathrm{g})$, tobramycin $(10 \mu \mathrm{g})$, gentamicin $(10 \mu \mathrm{g})$, ceftazidime $(10 \mu \mathrm{g})$, cefepime $(30 \mu \mathrm{g})$, imipenem $(10 \mu \mathrm{g})$, meropenem $(10 \mu \mathrm{g})$, levofloxacin $(5 \mu \mathrm{g})$, piperacillin $(30 \mu \mathrm{g})$, piperacillin / tazobactam $(30 / 6 \mu \mathrm{g})$, ticarcillin / clavulanic acid (75/10 $\mu \mathrm{g}$ ) (all Emapol antibiotic discs). The cultivation was carried out for $18 \pm 2 \mathrm{~h}$ at $35^{\circ} \mathrm{C} \pm 1{ }^{\circ} \mathrm{C}$ under aerobic conditions. The results were interpreted following the current recommendations of the European Committee on Antimicrobial Susceptibility Testing (EUCAST) [40].

\subsection{Extraction and Samples Preparation \\ 5.3.1. Intracellular Metabolites}

$20 \mathrm{mg}$ of lyophilized cells were suspended in $600 \mu \mathrm{L}$ of methanol and samples were disrupted for $5 \mathrm{~min}$ in TissueLyser (Tissue Lyser II, Qiagen, Venlo, Netherlands). Then $600 \mu \mathrm{L}$ of water was added to each sample and again vortexed for $10 \mathrm{~min}$. After the disintegration, samples were centrifuged for $10 \mathrm{~min}$, at $12000 \mathrm{rpm}$ at $4{ }^{\circ} \mathrm{C}$ (Micro 220R, Hettich), and $0.9 \mathrm{~mL}$ of clarified upper phase was transferred into a new tube. The extracts were evaporated in a vacuum centrifuge (WP-03, JW Electronic, United States) $\left(40{ }^{\circ} \mathrm{C}, 1500 \mathrm{rpm}, 10 \mathrm{~h}\right)$. In the next step, $600 \mu \mathrm{L}$ of PBS buffer $\left(0.1 \mathrm{M}, 10 \% \mathrm{D}_{2} \mathrm{O}, \mathrm{pH}=7.0\right.$, TSP $=0.3 \mathrm{mM}$ ) was added to each sample and mixed for $1 \mathrm{~min}$ and $550 \mu \mathrm{L}$ was transferred into NMR-tubes (SP, 5mm, Armar Chemicals, Germany) for measurements. Until the measurements were taken, the samples were stored at $4{ }^{\circ} \mathrm{C}$.

\subsubsection{Extracellular Metabolites}

After breeding, $1.5 \mathrm{~mL}$ medium were evaporated in a vacuum centrifuge $\left(40{ }^{\circ} \mathrm{C}\right.$, $1500 \mathrm{rpm}, 12 \mathrm{~h})$. In the next step, $600 \mu \mathrm{L}$ of PBS buffer $\left(0.1 \mathrm{M}\left(\mathrm{NaH}_{2} \mathrm{PO}_{4} / \mathrm{Na}_{2} \mathrm{HPO}_{4}\right), 10 \%\right.$ $\mathrm{D}_{2} \mathrm{O}, \mathrm{pH}=7.0, \mathrm{TSP}=0.5 \mathrm{mM}$ ) was added to each sample and mixed for $3 \mathrm{~min}$ and $550 \mu \mathrm{L}$ was transferred into 5-mm NMR-tubes (5SP, Armar Chemicals, Germany) for measurements. Until the measurements were taken, the samples were stored at $4{ }^{\circ} \mathrm{C}$.

\section{4. ${ }^{1} \mathrm{H}$ NMR Spectroscopy Analysis of the Bacterial Metabolites}

Standard ${ }^{1} \mathrm{H}$ NMR experiments were performed on a Bruker AVANCE II $600.58 \mathrm{MHz}$ spectrometer (Bruker, GmBH, Germany) equipped with a $5 \mathrm{~mm}$ TBO probe at $298 \mathrm{~K}$. All one-dimensional ${ }^{1} \mathrm{H}$ NMR spectra were carried out using the cpmgpr1d (in Bruker notation) pulse sequence by suppression of water resonance by presaturation. Acquisition parameters were as follows: spectral width, $10 \mathrm{ppm}$; the number of scans, 128; acquisition time, $2.72 \mathrm{~s}$ per scan; relaxation delay, $3.5 \mathrm{~s}$; and time-domain points, $64 \mathrm{~K}$. The spectra were referenced to the TSP resonance at $0.0 \mathrm{ppm}$ and manually corrected for phase and baseline (MestReNova v. 11.0.3, Qingdao, China).

\subsection{Data Processing and Multivariate Statistical Data Analysis}

All spectra were exported to Matlab (Matlab R2014a, v. 8.3.0.532, Natick, MA, USA) for preprocessing. Regions affected by solvent suppression were excluded (4.55-5.10 ppm for intracellular analysis and $4.58-4.90 \mathrm{ppm}$ for extracellular analysis) and alignment procedures involving the correlation of optimized warping (COW) and interval correlation shifting (icoshift) algorithms were applied [41,42]. The spectra consisted of 8910 data points and were normalized using the probabilistic quotient method to overcome the issue of dilution [43].

The multivariate and statistical data analysis was performed on a set of the 32 assigned metabolites for intracellular metabolites and 27 metabolites for extracellular metabolites. All assignments were verified using the following databases: KEGG Pathways, PubChem, PAMDB, and ChenomX software (Chenomx Inc., Edmonton, AB, Canada). The concen- 
tration of metabolite measured by NMR was obtained as the sum of the intensities of the no overlapping resonances (or a part of partly overlapping resonances). The input for SIMCA-P software was a transformed data matrix (v 15.02, Umetrics, Umeå, Sweden). For additional analysis, Matlab was used. The data sets were unit variance scaled before the chemometric analysis. For bacteria strain classification, principal component analysis (PCA), and partial least square analysis (OPLS) were carried out. The multivariate data visualization marked an ellipse with Hotelling's T2 range (95\%). The OPLS-DA model reliability was tested with CV-ANOVA at the level of significance of $\alpha<0.05$. The most important variable was discrimination between comparisons, which was selected based on the variable importance in projection (VIP) value with a cutoff value of 1.00. Univariate analysis was performed using MATLAB software with Student's $t$-test (equal/unequal variance) for data originating from a normal distribution and using Mann-Whitney-Wilcoxon test for data that did not meet this requirement. Normality of distribution was assessed by the Shapiro-Wilk test. The correction for multiple comparisons was preceded by the Benjamini-Hochberg procedure (FDR). All univariate statistics were carried out at the level of significance of $\alpha<0.05$.

\subsection{Bioinformatics Analysis}

After preprocessing, both, extra- and intracellular metabolite sets from two phenotypically different strains of Pseudomonas aeruginosa (antibiotic-resistant strain and antibioticsusceptible strain) were used to perform metabolic pathway enrichment in the FELLA package [26]. FELLA is an R-package (public software) available in under the GPL-3 license [44]. To perform pathways, metabolites with statistical importance and VIP value $>1.00$ were used. Firstly, KEGG-based hierarchical representations of biochemistry (knowledge graph) were built using P. aeruginosa PAO1 (T00035, Release 97.0+/03-04, Mar 21). Later, a list of metabolites from examined strains was separately mapped to the internal representation, creating an enriched object, and then subsequently the propagation algorithm was run using the diffusion method (undirected heat diffusion model) to score graph nodes. Additionally, the parametric z-score was computed using normality approximations for statistical normalization.

Supplementary Materials: The following are available online at https: / www.mdpi.com/article/10 .3390/ijms221910820/s1. Table S1: ${ }^{1} \mathrm{H}$ NMR signal assignments for intracellular metabolites. Table S2: ${ }^{1} \mathrm{H}$ NMR signal assignments for extracellular metabolites. Figure S1: OPLS-DA score plot of ${ }^{1} \mathrm{H}$ NMR data of P. aeruginosa strains. Drug-resistant extracellular (green), drug-susceptible extracellular (yellow), drug-resistant intracellular (blue), drug-susceptible intracellular (red). Figure S2: OPLSDA score plot of ${ }^{1} \mathrm{H}$ NMR data of intracellular metabolites of drug-resistant (blue squares) and drug-susceptible (red circle) P. aeruginosa strains. Figure S3: OPLS-DA models for ${ }^{1} \mathrm{H}$ NMR data of extracellular metabolites of $P$. aeruginosa strains. (A) both P. aeruginosa strains with control; (B) both P. aeruginosa strains; (C) drug-resistant $P$. aeruginosa strain with control; (D) drug-susceptible $P$. aeruginosa strain with control. (drug-resistant extracellular (green squares), drug- susceptible extracellular (yellow circle), control-LB medium (gray triangles)). Figure S4: PCA model plots and corresponding loading plot for ${ }^{1} \mathrm{H}$ NMR data of extracellular metabolites of $P$. aeruginosa strains. (A) drug-resistant P. aeruginosa strain with control (the first and second principal component (PC) accounted respectively for $67.6 \%$ and $11.8 \%$ of the total variance in the data $(\mathrm{R} 2 \mathrm{X}=0.864)$ ); (B) drugsusceptible $P$. aeruginosa strain with control (the first and second principal component (PC) accounted respectively for $69.9 \%$ and $14.5 \%$ of the total variance in the data $(\mathrm{R} 2 \mathrm{X}=0.845)$ ), (drug-resistant extracellular (green squares), drug- susceptible extracellular (yellow circle), control—LB medium (gray triangles)). File S1: Interactive version of results of the node prioritization by FELLA in the Pseudomonas aeruginosa strain (intracellular analysis). File S2: Interactive version of results of the node prioritization by FELLA in the Pseudomonas aeruginosa strain (extracellular analysis).

Author Contributions: Conceptualization. K.A.M. and S.J.J.; Software. K.A.M. and Ł.P.; methodology. K.A.M. and S.J.J.; validation. S.J.J., M.Ł. and P.M.; formal analysis. K.A.M.; investigation. K.A.M. and S.J.J.; resources. D.S. and J.M.; writing—original draft preparation. K.A.M. and J.M.; 
writing-review and editing. M.Ł. and P.M.; visualization. K.A.M. and Ł.P.; supervision. M.Ł and P.M. All authors have read and agreed to the published version of the manuscript.

Funding: Statutory project of WUST; Faculty of Chemistry; Department of Biochemistry, Molecular Biology and Biotechnology (2021).

Institutional Review Board Statement: Not applicable.

Informed Consent Statement: Not applicable.

Data Availability Statement: MDPI Research Data Policies.

Conflicts of Interest: The authors declare no conflict of interest.

\section{References}

1. Paterson, D.L.; Kim, B.N. Pseudomonas aeruginosa. In Antimicrobial Drug Resistance; Humana Press: Totowa, NJ, USA, 2009; pp. 811-817.

2. Ciofu, O.; Tolker-Nielsen, T. Tolerance and Resistance of Pseudomonas aeruginosa Biofilms to Antimicrobial Agents-How P. aeruginosa Can Escape Antibiotics. Front. Microbiol. 2019, 10, 913. [CrossRef]

3. Behzadi, P.; Barath, Z.; Gajdacs, M. It's not easy being green: A narrative review on the microbiology, virulence and therapeutic prospects of mlutidrag-resistant Pseudomonas aeruginosa. Antibiotics 2021, 10, 42. [CrossRef]

4. Bonomo, R.A.; Szabo, D. Mechanisms of multidrug resistance in Acinetobacter species and Pseudomonas aeruginosa. Clin. Infect. Dis. 2006, 43, 49-56. [CrossRef] [PubMed]

5. WHO Report. Available online: https://www.who.int/news/item/27-02-2017-who-publishes-list-of-bacteria-for-which-newantibiotics-are-urgently-needed (accessed on 20 May 2021).

6. De Oliveira, D.M.P.; Forde, B.M.; Kidd, T.J.; Harris, P.N.A.; Schembri, M.A.; Beatson, S.A.; Paterson, D.L.; Walker, M.J. Antimicrobial resistance in ESKAPE pathogens. Clin. Microbiol. Rev. 2020, 33. [CrossRef]

7. Tiwari, V. Post-translational modification of ESKAPE pathogens as a potential target in drug discovery. Drug Discov. Today 2019, 24, 814-822. [CrossRef] [PubMed]

8. Santajit, S.; Indrawattana, N. Mechanisms of Antimicrobial Resistance in ESKAPE Pathogens. Biomed Res. Int. 2016, $2016,2475067$. [CrossRef] [PubMed]

9. Morita, Y.; Tomida, J.; Kawamura, Y. Responses of Pseudomonas aeruginosa to antimicrobials. Front. Microbiol. 2014, 4, 1-8. [CrossRef]

10. Livermore, D.M. Of Pseudomonas, porins, pumps and carbapenems. J. Antimicrob. Chemother. 2001, 47, 247-250. [CrossRef] [PubMed]

11. Lambert, P.A. Bacterial resistance to antibiotics: Modified target sites. Adv. Drug Deliv. Rev. 2005, 57, 1471-1485. [CrossRef]

12. Grajdacs, M. Carbapenem-resistance but cephalosporin susceptible Pseudomonas aeruginosa in urinary tract infections: Opportunity for colistin sparing. Antibiotics 2020, 9, 153. [CrossRef]

13. Ishida, H.; Ishida, Y.; Kurosaka, Y.; Otani, T.; Sato, K.; Kobayashi, H. In Vitro and In Vivo Activities of Levofloxacin against Biofilm-Producing Pseudomonas aeruginosa. Antimicrob. Agents Chemother. 1998, 42, 1641-1645. [CrossRef]

14. Meylan, S.; Porter, C.B.M.; Yang, J.H.; Belenky, P.; Gutierrez, A.; Lobritz, M.A.; Park, J.; Kim, S.H.; Moskowitz, S.M.; Collins, J.J. Carbon Sources Tune Antibiotic Susceptibility in Pseudomonas aeruginosa via Tricarboxylic Acid Cycle Control. Cell Chem. Biol. 2017, 24, 195-206. [CrossRef]

15. Nguyen, D.; Joshi-Datar, A.; Lepine, F.; Bauerle, E.; Olakanmi, O.; Beer, K.; McKay, G.; Siehnel, R.; Schafhauser, J.; Wang, Y.; et al. Active Starvation Responses Mediate Antibiotic Tolerance in Biofilms and Nutrient-Limited Bacteria. Science 2011, 334, $982-986$. [CrossRef] [PubMed]

16. Aldridge, B.B.; Rhee, K.Y. Microbial metabolomics: Innovation, application, insight. Curr. Opin. Microbiol. $2014,19,90-96$. [CrossRef] [PubMed]

17. Tang, J. Microbial Metabolomics. Curr. Genomics 2011, 12, 391-403. [CrossRef]

18. Patti, G.J.; Yanes, O.; Siuzdak, G. Metabolomics: The apogee of the omics trilogy. Nat. Rev. Mol. Cell Biol. 2012, 13, 263-269. [CrossRef]

19. Mielko, K.A.; Jabłoński, S.J.; Wojtowicz, W.; Milczewska, J.; Sands, D.; Łukaszewicz, M.; Młynarz, P. Possible metabolic switch between environmental and pathogenic Pseudomonas aeruginosa strains: 1H NMR based metabolomics study. J. Pharm. Biomed. Anal. 2020, 188, 113369. [CrossRef] [PubMed]

20. Welker, M.; Van Belkum, A.; Girard, V.; Charrier, J.P.; Pincus, D. An update on the routine application of MALDI-TOF MS in clinical microbiology. Expert Rev. Proteom. 2019, 16, 695-710. [CrossRef] [PubMed]

21. Palama, T.L.; Canard, I.; Rautureau, G.J.P.; Mirande, C.; Chatellier, S.; Elena-Herrmann, B. Identification of bacterial species by untargeted NMR spectroscopy of the exo-metabolome. Analyst 2016, 141, 4558-4561. [CrossRef]

22. Zhao, X.; Shen, M.; Jiang, X.; Shen, W.; Zhong, Q.; Yang, Y.; Tan, Y.; Agnello, M.; He, X.; Hu, F.; et al. Transcriptomic and Metabolomics Profiling of Phage-Host Interactions between Phage PaP1 and Pseudomonas aeruginosa. Front. Microbiol. 2017, 8 , 1-10. [CrossRef] 
23. Han, M.-L.; Zhu, Y.; Creek, D.J.; Lin, Y.-W.; Anderson, D.; Shen, H.-H.; Tsuji, B.; Gutu, A.D.; Moskowitz, S.M.; Velkov, T.; et al. Alterations of Metabolic and Lipid Profiles in Polymyxin-Resistant Pseudomonas aeruginosa. Antimicrob. Agents Chemother. 2018, 62. [CrossRef]

24. Gupta, A.; Bansal, N.; Houston, B. Metabolomics of urinary tract infection: A new uroscope in town. Expert Rev. Mol. Diagn. 2012, 12, 361-369. [CrossRef]

25. Traxler, M.F.; Summers, S.M.; Nguyen, H.-T.; Zacharia, V.M.; Hightower, G.A.; Smith, J.T.; Conway, T. The global, ppGpp-mediated stringent response to amino acid starvation in Escherichia coli. Mol. Microbiol. 2008, 68, 1128-1148. [CrossRef]

26. Picart-Armada, S.; Fernández-Albert, F.; Vinaixa, M.; Yanes, O.; Perera-Lluna, A. FELLA: An R package to enrich metabolomics data. BMC Bioinform. 2018, 19, 538. [CrossRef]

27. Guo, Q.; Wei, Y.; Xia, B.; Jin, Y.; Liu, C.; Pan, X.; Shi, J.; Zhu, F.; Li, J.; Qian, L.; et al. Identification of a small molecule that simultaneously suppresses virulence and antibiotic resistance of Pseudomonas aeruginosa. Sci. Rep. 2016, 6, 1-15. [CrossRef]

28. La Rosa, R.; Johansen, H.K.; Molin, S. Adapting to the Airways: Metabolic Requirements of Pseudomonas aeruginosa during the Infection of Cystic Fibrosis Patients. Metabolites 2019, 9, 234. [CrossRef]

29. Nicholson, J.K.; Wilson, I.D. Understanding "global" systems biology: Metabonomics and the continuum of metabolism. Nat. Rev. Drug Discov. 2003, 2, 668-676. [CrossRef]

30. Wang, Y.; Han, B.; Xie, Y.; Wang, H.; Wang, R.; Xia, W.; Li, H.; Sun, H. Combination of gallium III with acetate for combating antibiotic resistant Pseudomonas aeruginosa. Chem. Sci. 2019, 10, 6099-6106. [CrossRef]

31. Palmer, K.L.; Aye, L.M.; Whiteley, M. Nutritional cues control Pseudomonas aeruginosa multicellular behavior in cystic fibrosis sputum. J. Bacteriol. 2007, 189, 8079-8087. [CrossRef]

32. Amorim Franco, T.M.; Blanchard, J.S. Bacterial Branched-Chain Amino Acid Biosynthesis: Structures, Mechanisms, and Drugability. Biochemistry 2017, 56, 5849-5865. [CrossRef]

33. Aliashkevich, A.; Alvarez, L.; Cava, F. New insights into the mechanisms and biological roles of D-amino acids in complex eco-systems. Front. Microbiol. 2018, 9, 683. [CrossRef]

34. Behrends, V.; Ryall, B.; Zlosnik, J.E.A.; Speert, D.P.; Bundy, J.G.; Williams, H.D. Metabolic adaptations of Pseudomonas aeruginosa during cystic fibrosis chronic lung infections. Environ. Microbiol. 2013. [CrossRef]

35. Liu, Y.; Yang, K.; Zhang, H.; Jia, Y.; Wang, Z. Combating Antibiotic Tolerance Through Activating Bacterial Metabolism. Front. Microbiol. 2020, 11, 577564. [CrossRef]

36. Kanehisa, M.; Furumichi, M.; Tanabe, M.; Sato, Y.; Morishima, K. KEGG: New perspectives on genomes, pathways, diseases and drugs. Nucleic Acids Res. 2017, 45, D353-D361. [CrossRef]

37. Radkov, A.D.; Moe, L.A. Bacterial synthesis of d-amino acids. Appl. Microbiol. Biotechnol. 2014, 98, 5363-5374. [CrossRef]

38. Nyyssölä, A.; Reinikainen, T.; Leisola, M. Characterization of Glycine Sarcosine N -Methyltransferase and Sarcosine Dimethylglycine N-Methyltransferase. Appl. Environ. Microbiol. 2001, 67, 2044-2050. [CrossRef]

39. Favia, M.; Gallo, C.; Guerra, L.; De Venuto, D.; Diana, A.; Polizzi, A.M.; Montemurro, P.; Mariggiò, M.A.; Leonetti, G.; Manca, A.; et al. Treatment of Cystic Fibrosis Patients Homozygous for F508del with Lumacaftor-Ivacaftor (Orkambi®) Restores Defective CFTR Channel Function in Circulating Mononuclear Cells. Int. J. Mol. Sci. 2020, 21, 2398. [CrossRef]

40. Biemer, J.J. Antimicrobial susceptibility testing by the Kirby-Bauer disc diffusion method. Ann. Clin. Lab. Sci. 1973, 3, 135-140.

41. Savorani, F.; Tomasi, G.; Engelsen, S.B. icoshift: A versatile tool for the rapid alignment of 1D NMR spectra. J. Magn. Reson. 2010, 202, 190-202. [CrossRef]

42. Tomasi, G.; van den Berg, F.; Andersson, C. Correlation optimized warping and dynamic time warping as preprocessing methods for chromatographic data. J. Chemom. 2004, 18, 231-241. [CrossRef]

43. Dieterle, F.; Ross, A.; Schlotterbeck, G.; Senn, H.; Hoffman, F. Probabilistic Quotient Normalization as Robust Method to Account for Dilution of Complex Biological Mixtures. Application in $1 \mathrm{H}$ NMR Metabonomics. Anal. Chem. 2006. [CrossRef] [PubMed]

44. FELLA in R-package. Available online: https:/ / github.com/b2slab/FELLA (accessed on 23 September 2021). 